\title{
Capacity of Channels with Action-Dependent States
}

\author{
Tsachy Weissman*
}

January 20, 2009

\begin{abstract}
We consider channels with action-dependent states: Given the message to be communicated, the transmitter chooses an action sequence that affects the formation of the channel states, and then creates the channel input sequence based on the state sequence. We characterize the capacity of such a channel both for the case where the channel inputs are allowed to depend non-causally on the state sequence and the case where they are restricted to causal dependence. Our setting covers previously considered scenarios involving transmission over channels with states known at the encoder, as well as various new coding scenarios for channels with a 'rewrite' option that may arise naturally in storage for computer memories with defects or in magnetic recoding. A few examples are worked out in detail.
\end{abstract}

Key words and phrases: Actions, Channel with a Rewrite option, Channel with states, Cost constraints, Dirty paper coding, Gel'fand-Pinsker Channel, Shannon Channel.

\section{Introduction}

Communication through state-dependent channels, with states known at the transmitter, is a problem that has received much attention since the work of Shannon [11], Kusnetsov and Tsybakov [7], Gel'fand and Pinsker [4], and Heegard and El Gamal [5]. The assumption in these seminal papers, as well as in the work on communication with state-dependent channels that followed (cf. [6] and references therein), is that the channel states are generated by nature, and cannot be affected or controlled by the communication system.

In this work, we revisit this problem setting for the case where the transmitter can take actions that affect the formation of the states. Specifically, we consider a communication system where encoding is in two parts: given the message, an action sequence is created. The actions affect the formation of the channel states, which are accessible to the transmitter when producing the channel input sequence. A channel with action-dependent states then is characterized by two ingredients: the distribution of state given an action $P_{S \mid A}$ (in lieu of the distribution of the state $P_{S}$ in the original setting) and, as in the original setting, the distribution of the channel output given the input and state $P_{Y \mid X, S}$. We characterize the capacity of such a channel both for the case where the channel inputs are allowed to depend non-causally on the state sequence, and that where they are restricted to causal dependence. Our problem can be thought of as the channel coding dual of source coding, with decoder side information, where the decoder is allowed to choose actions that affect the nature and quality of the side information, as considered in [9].

Beyond merely generalizing previously considered problems involving coding with states known at the transmitter, our framework captures various new channel coding scenarios that may arise naturally in recording for magnetic storage devices or coding for computer memories with defects. Concretely, consider a 2 -stage procedure for recording on a memory with defects. After writing into the memory for the first time, the encoder observes a noisy version of

*Department of Electrical Engineering, Technion, Haifa 32000, Israel. On leave from Department of Electrical Engineering, Stanford University, Stanford, CA 94305, USA. Email: tsachy@ee.technion.ac.il 
what the decoder will see when it tries to read from the memory. The encoder is now allowed to rewrite at whichever memory locations it chooses before the decoder attempts to decipher the information. How much information can be reliably communicated in this process? Suppose that in the first use of the memory neither encoder nor decoder know where the defects are. Then, in the second use (the 'rewrite' stage), the encoder will have some idea on these defects according to the signal he input in the first stage and the noisy measurement of the channel output for that stage. In general, there is a tension between the amount of information the encoder can convey in the first pass and its ability to learn about the channel state to better communicate in the second pass. Our framework quantifies this tension and yields a characterization of the fundamental limits on communication for such 2-stage coding systems.

The remainder of the paper is organized as follows: Sections 2 and 3 are dedicated, respectively, to characterizing the fundamental limits of communication over channels with action-dependent states available at the encoder, when the states are available non-causally and causally. In Section 4 we extend our results to the case of cost constraints, point out equivalent representations of our capacity formulae, and discuss some special cases. In Section 5 we apply our results to characterize the capacity for various coding scenarios involving a channel 'rewrite' option. In Section 6 we look at a "writing-on-clean-paper-and-then-writing-on-its-corrupted version" channel, which is an extension of Costa's dirty paper problem [1] to our setting. We conclude in Section 7 with a summary of our work and some future directions.

\section{Non-Causally Available States}

Let upper case, lower case, and calligraphic letters denote, respectively, random variables, specific or deterministic values they may assume, and their alphabets. For two jointly distributed random objects $X$ and $Y$, let $P_{X}, P_{X, Y}$, and $P_{X \mid Y}$ respectively denote the distribution of $X$, the joint distribution of $X, Y$, and the conditional distribution of $X$ given $Y$. In particular, when $X$ and $Y$ are discrete, $P_{X \mid Y}$ represents the stochastic matrix whose elements are $P_{X \mid Y}(x \mid y)=P(X=x \mid Y=y) . X_{m}^{n}$ denotes the $n-m+1$-tuple $\left(X_{m}, \ldots, X_{n}\right)$ when $m \leq n$ and the empty set otherwise. $X^{n}$ is shorthand for $X_{1}^{n}$.

We dedicate this section to characterizing the fundamental limits on reliable communication for schemes of the form depicted in Figure 1: given the message $M$, selected uniformly at random from the message set $\mathcal{M}=$ $\{1, \ldots,|\mathcal{M}|\}$, an action sequence $A^{n}=A^{n}(M)$ is selected. Nature now generates the state sequence $S^{n}$ as the output of the memoryless channel $P_{S \mid A}$ whose input is $A^{n}$. A channel input sequence is now selected on the basis of the message and the whole state sequence $X^{n}=X^{n}\left(M, S^{n}\right)$. The joint PMF of $M, A^{n}, S^{n}, X^{n}, Y^{n}$, induced by a given scheme, is thus

$$
P_{M, A^{n}, S^{n}, X^{n}, Y^{n}}\left(m, a^{n}, s^{n}, x^{n}, y^{n}\right)=\frac{1_{\left\{A^{n}(m)=a^{n}, X^{n}\left(m, s^{n}\right)=x^{n}\right\}}}{|\mathcal{M}|} \prod_{i=1}^{n} P_{S \mid A}\left(s_{i} \mid a_{i}\right) P_{Y \mid X, S}\left(y_{i} \mid x_{i}, s_{i}\right) .
$$

The associated probability of error is $P_{e}=P\left(M \neq \hat{M}\left(Y^{n}\right)\right)$, where $\hat{M}\left(Y^{n}\right)$ is the best (maximum likelihood) estimate of $M$ based on $Y^{n}$ under the joint distribution in (1). The rate $R$ is said to be achievable if there exists a sequence of schemes for increasing block lengths with $\frac{1}{n} \log |\mathcal{M}| \leq R$ and $P_{e} \stackrel{n \rightarrow \infty}{\longrightarrow} 0$. The capacity of the channel with action-dependent states known non-causally to the transmitter is the supremum over all achievable rates.

Theorem 1 The capacity of the channel with action-dependent states known non-causally to the transmitter is given by

$$
C=\max [I(U ; Y)-I(U ; S \mid A)]
$$




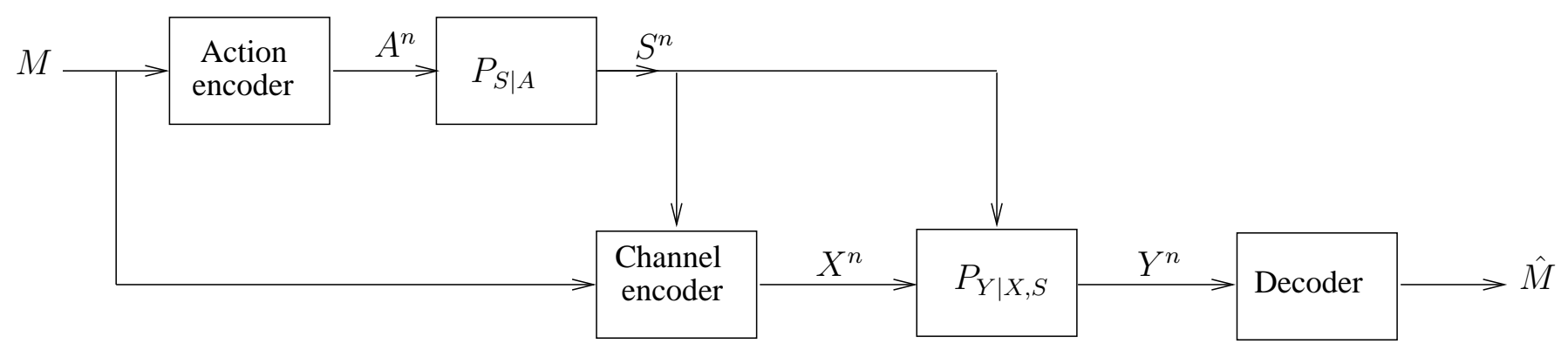

Figure 1: Channel with action-dependent states.

where the maximization is over all joint distributions of the form

$$
P_{A, S, U, X, Y}(a, s, u, x, y)=P_{A}(a) P_{S \mid A}(s \mid a) P_{U \mid S, A}(u \mid s, a) 1_{\{x=f(u, s)\}} P_{Y \mid X, S}(y \mid x, s)
$$

for some $P_{A}, P_{U \mid S, A}, f$ and $|\mathcal{U}| \leq|\mathcal{A}||\mathcal{S}||\mathcal{X}|+1$.

Comments:

- Similarly as in the classical Gelfand-Pinsker setting [4], the maximum in (2) does not increase when allowing more general distributions of the form

$$
P_{A, S, U, X, Y}(a, s, u, x, y)=P_{A}(a) P_{S \mid A}(s \mid a) P_{U \mid S, A}(u \mid s, a) P_{X \mid U, S}(x \mid u, s) P_{Y \mid X, S}(y \mid x, s),
$$

i.e., allowing a general conditional distribution $P_{X \mid U, S}$ rather than restricting $X$ to be a deterministic function of $(U, S)$ as in (3). The reason is that $I(U ; Y)-I(U ; S \mid A)$ is a convex functional of $P_{X \mid U, S}$ (when all other distributions and conditional distributions in (3) are fixed), and thus maximized at a corner point of the simplex. To see why this convexity holds note that $I(U ; S \mid A)$ depends only on $P_{U, S, A}$ so we need only establish convexity of $I(U ; Y)$ in $P_{X \mid U, S}$. To this end take two conditional distributions $P_{X \mid U, S}^{(1)}, P_{X \mid U, S}^{(2)}$ and let $P_{X \mid U, S}=$ $\alpha P_{X \mid U, S}^{(1)}+(1-\alpha) P_{X \mid U, S}^{(2)}$ for $\alpha \in[0,1]$. The convexity of $I(U ; Y)$ in $P_{X \mid U, S}$ follows by the convexity of $I(U ; Y)$ in $P_{Y \mid U}$ (cf., e.g., [2]) upon noting that $P_{Y \mid U}=\alpha P_{Y \mid U}^{(1)}+(1-\alpha) P_{Y \mid U}^{(2)}$ when $P_{Y \mid U}, P_{Y \mid U}^{(1)}, P_{Y \mid U}^{(2)}$ denote the conditional distributions induced, respectively, by $P_{X \mid U, S}, P_{X \mid U, S}^{(1)}, P_{X \mid U, S}^{(2)}$.

- Let $C_{G P}\left(P_{S}, P_{Y \mid X, S}\right)$ denote the capacity of the channel with states known non-causally at the transmitter, as considered in $[4,5]$. It is natural to wonder how the capacity in our setting, as characterized in Theorem 1 , compares with $\max _{a \in \mathcal{A}} C_{G P}\left(P_{S \mid A=a}, P_{Y \mid X, S}\right)$, the rate that would be achieved by greedily selecting the action leading to the best Gelfand-Pinsker (GP) channel at all time points, and proceeding with an optimal GP code for that channel. Under such a strategy, no information is conveyed by the actions which are only used to set up the channel, and communication is performed only at the second stage. For an extreme example of how suboptimal such a strategy can be, consider a channel for which $P_{Y \mid X, S}=P_{Y \mid S}$. Clearly $C_{G P}\left(P_{S}, P_{Y \mid X, S}\right)=0$ for any such channel and therefore $\max _{a \in \mathcal{A}} C_{G P}\left(P_{S \mid A=a}, P_{Y \mid X, S}\right)=0$. On the other hand, as is readily seen to follow by applying Theorem 1 or from first principles, the capacity of this channel is $\max _{P_{A}} I(A ; Y)$, which may be positive. More generally, the capacity achieving scheme finds the optimal balance between conveying information through the choice of actions and the tendency to take actions that will result in states conducive for the communication in the second stage. 
Proof of Theorem 1:

Proof of Achievability: Fix $P_{A}, P_{U \mid S, A}, f$ and consider $A, S, U, X, Y$ jointly distributed as in (3).

- Generate ${ }^{1}\left\{A^{n}(m)\right\}_{m=1}^{2^{n R}} n$-tuples iid $\sim P_{A}$

- For each $1 \leq m \leq 2^{n R}$ generate $\left\{U^{n}(j, m)\right\}_{j=1}^{2^{n R^{\prime}}}$ iid $\sim \prod_{i=1}^{n} P_{U \mid A}\left(\cdot \mid A_{i}(m)\right)$

- Encoding:

- choose $A^{n}(M)$ as the action sequence

- let $S^{n}$ be the state sequence generated in response to the action sequence

- let $J$ be the smallest value of $j$ such that $\left(U^{n}(j, M), S^{n}, A^{n}(M)\right) \in T_{P},{ }^{2}$ and take $J=1$ if no such $j$ exists

- let the channel input sequence be given by $X^{n}=f\left(U^{n}(J, M), S^{n}\right)$, where $f\left(U^{n}, S^{n}\right)$ denotes the $n$-tuple whose $i$ th component is $f\left(U_{i}, S_{i}\right)$

- Decoding:

- seeing the channel output $Y^{n}$, let $\hat{M}$ be the smallest value of $\hat{m}$ for which there exists a $\hat{j}$ such that $\left(A^{n}(\hat{m}), U^{n}(\hat{j}, \hat{m}), Y^{n}\right) \in T_{P}$, and take $\hat{M}=1$ if no such $\hat{m}$ exists.

The event $M \neq \hat{M}$ is contained in the union of the following three error events:

- At the encoding stage, there exists no $j$ such that $\left(U^{n}(j, M), S^{n}, A^{n}(M)\right) \in T_{P}$. The probability of this event is vanishing so long as $R^{\prime}>I(U ; S \mid A)$.

- There exists $\hat{m} \neq M$ for which there exists a $\hat{j}$ such that $\left(A^{n}(\hat{m}), U^{n}(\hat{j}, \hat{m}), Y^{n}\right) \in T_{P}$. To bound the probability of this event note first that, for all $\hat{m}$ and $\hat{j}$,

$$
P\left(\left(A^{n}(\hat{m}), U^{n}(\hat{j}, \hat{m}), Y^{n}\right) \in T_{P} \mid \hat{m} \neq M\right) \doteq 2^{-n I(A, U ; Y)} \leq 2^{-n I(U ; Y)} .
$$

Therefore, by the union bound,

$$
P\left(\cup_{\hat{j}}\left\{\left(A^{n}(\hat{m}), U^{n}(\hat{j}, \hat{m}), Y^{n}\right) \in T_{P}\right\} \mid \hat{m} \neq M\right) \leq 2^{-n\left(I(U ; Y)-R^{\prime}\right)} .
$$

It follows that the probability of the existence of $\hat{m} \neq M$ for which there exists a $\hat{j}$ such that $\left(A^{n}(\hat{m}), U^{n}(\hat{j}, \hat{m}), Y^{n}\right) \in$ $T_{P}$ is vanishing provided $R<I(U ; Y)-R^{\prime}$.

- $\left(A^{n}(M), U^{n}(j, M), Y^{n}\right) \notin T_{P}$ for all $j$. The probability of this event is vanishing so long as $R^{\prime}>I(U ; S \mid A)$ since, as argued for the first event, $\left(U^{n}(J, M), S^{n}, A^{n}(M)\right) \in T_{P}$ with probability approaching one and, hence, so is the probability that $\left(U^{n}(J, M), S^{n}, A^{n}(M), X^{n}, Y^{n}\right) \in T_{P}$.

Thus we have established the existence of a sequence of schemes with $R^{\prime}=I(U ; S \mid A)+\varepsilon, R=I(U ; Y)-I(U ; S \mid A)-2 \varepsilon$, and vanishing probability of decoding error.

\footnotetext{
${ }^{1}$ Here and throughout we will ignore integer constraints, writing $2^{n R}$ in lieu of the more precise $\left\lfloor 2^{n R}\right\rfloor$.

${ }^{2}$ To avoid cumbersome notation, we let $T_{P}$ generically denote sets that are typical in the sense of [3] (cf., in particular, the $\delta$-convention therein), with respect to (joint) distributions that are clear from the context.
} 
Proof of Converse: Fix a scheme and consider:

$$
\begin{aligned}
& I\left(M ; Y^{n}\right) \\
= & I\left(M ; Y^{n}\right)-I\left(M ; S^{n} \mid A^{n}\right) \\
= & \sum_{i=1}^{n} I\left(M ; Y_{i} \mid Y^{i-1}\right)-I\left(M ; S_{i} \mid S_{i+1}^{n}, A^{n}\right) \\
= & \sum_{i=1}^{n} I\left(M, S_{i+1}^{n}, A^{n} ; Y_{i} \mid Y^{i-1}\right)-I\left(S_{i+1}^{n}, A^{n} ; Y_{i} \mid M, Y^{i-1}\right)-I\left(M, Y^{i-1} ; S_{i} \mid S_{i+1}^{n}, A^{n}\right)+I\left(Y^{i-1} ; S_{i}, A^{n} \mid M, S_{i+1}^{n}, A^{n}\right) \\
= & \sum_{i=1}^{n} I\left(M, S_{i+1}^{n}, A^{n} ; Y_{i} \mid Y^{i-1}\right)-I\left(M, Y^{i-1} ; S_{i} \mid S_{i+1}^{n}, A^{n}\right) \\
\leq & \sum_{i=1}^{n} H\left(Y_{i}\right)-H\left(Y_{i} \mid Y^{i-1}, M, S_{i+1}^{n}, A^{n}\right)-\left[H\left(S_{i} \mid S_{i+1}^{n}, A^{n}\right)-H\left(S_{i} \mid Y^{i-1}, M, S_{i+1}^{n}, A^{n}\right)\right] \\
= & \sum_{i=1}^{n} H\left(Y_{i}\right)-H\left(Y_{i} \mid U_{i}\right)-\left[H\left(S_{i} \mid A_{i}\right)-H\left(S_{i} \mid U_{i}, A_{i}\right)\right] \\
= & \sum_{i=1}^{n} I\left(U_{i} ; Y_{i}\right)-I\left(U_{i} ; S_{i} \mid A_{i}\right) \\
\leq & n \max [I(U ; Y)-I(U ; S \mid A)]
\end{aligned}
$$

where:

- (6) is due to the Markov relation $M-A^{n}-S^{n}$

- (8) is due to the identities $I\left(M ; Y_{i} \mid Y^{i-1}\right)=I\left(M, S_{i+1}^{n}, A^{n} ; Y_{i} \mid Y^{i-1}\right)-I\left(S_{i+1}^{n}, A^{n} ; Y_{i} \mid M, Y^{i-1}\right)$ and $I\left(M ; S_{i} \mid S_{i+1}^{n}, A^{n}\right)=$ $I\left(M, Y^{i-1} ; S_{i} \mid S_{i+1}^{n}, A^{n}\right)-I\left(Y^{i-1} ; S_{i} \mid M, S_{i+1}^{n}, A^{n}\right)=I\left(M, Y^{i-1} ; S_{i} \mid S_{i+1}^{n}, A^{n}\right)-I\left(Y^{i-1} ; S_{i}, A^{n} \mid M, S_{i+1}^{n}, A^{n}\right)$

- (9) follows from the identity $\sum_{i=1}^{n} I\left(S_{i+1}^{n}, A^{n} ; Y_{i} \mid M, Y^{i-1}\right)=\sum_{i=1}^{n} I\left(Y^{i-1} ; S_{i}, A^{n} \mid M, S_{i+1}^{n}, A^{n}\right)$ which can be seen as follows:

$$
\begin{aligned}
\sum_{i=1}^{n} I\left(S_{i+1}^{n}, A^{n} ; Y_{i} \mid M, Y^{i-1}\right) & =\sum_{i=1}^{n-1} I\left(S_{i+1}^{n}, A^{n} ; Y_{i} \mid M, Y^{i-1}\right) \\
& =\sum_{i=1}^{n-1} \sum_{j=i+1}^{n} I\left(S_{j}, A^{n} ; Y_{i} \mid M, Y^{i-1}, S_{j+1}^{n}, A^{n}\right) \\
& =\sum_{j=1}^{n-1} \sum_{i=j+1}^{n} I\left(S_{i}, A^{n} ; Y_{j} \mid M, Y^{j-1}, S_{i+1}^{n}, A^{n}\right) \\
& =\sum_{i=2}^{n} \sum_{j=1}^{i-1} I\left(S_{i}, A^{n} ; Y_{j} \mid M, Y^{j-1}, S_{i+1}^{n}, A^{n}\right) \\
& =\sum_{i=2}^{n} I\left(S_{i}, A^{n} ; Y^{i-1} \mid M, S_{i+1}^{n}, A^{n}\right) \\
& =\sum_{i=1}^{n} I\left(S_{i}, A^{n} ; Y^{i-1} \mid M, S_{i+1}^{n}, A^{n}\right)
\end{aligned}
$$

- (11) is due to the Markov relation $S_{i}-A_{i}-\left(S_{i+1}^{n}, A^{n \backslash i}\right)$ and the definition $U_{i}=\left(M, Y^{i-1}, S_{i+1}^{n}, A^{n}\right)$ 
- the maximization in (13) is over distributions of the form in (3) for some $P_{A}, P_{U \mid S, A}, f$. That this maximum upper bounds each summand in (12) is due to the fact, noted following the statement of the theorem, about equivalence between maximization over distributions of the form in (3) and the form in (4), and the facts that for each $1 \leq i \leq n$ we have $P_{S_{i} \mid A_{i}}=P_{S \mid A}, P_{Y_{i} \mid X_{i}, S_{i}}=P_{Y \mid X, S}$ and the Markov relations $X_{i}-\left(U_{i}, S_{i}\right)-A_{i}$ and $\left(U_{i}, A_{i}\right)-\left(X_{i}, S_{i}\right)-Y_{i}$. The bound on the cardinality of $\mathcal{U}$ follows in a standard way via the support lemma of [3]: $\mathcal{U}$ should have $|\mathcal{A}||\mathcal{S}||\mathcal{X}|-1$ elements to preserve $P_{A, S, X}$ (which in turn preserves also $P_{A, S, X, Y}, H(Y$ ) and $H(S \mid A)$ ), plus two elements to preserve $H(Y \mid U)$ and $H(S \mid U, A)$.

The proof is completed via the usual appeal to Fano's inequality.

Comments:

- It is natural to wonder whether 'feedback' from the past states at the action stage might increase the capacity. In the proof of the converse part we have used the Markov relation $S_{i}-A_{i}-\left(S_{i+1}^{n}, A^{n \backslash i}\right)$, which need not hold when allowing actions of the form $A_{i}\left(M, S^{i-1}\right)$. Thus, our converse does not hold for that case and whether capacity could be increased when such dependence is allowed remains open.

- On the other hand, it is readily verified that all the Markov relations in the converse proof remain intact when the usual type of feedback is allowed, i.e., when $X_{i}$ is allowed to be of the form $X_{i}\left(M, S^{n}, Y^{i-1}\right)$. Evidently, similarly as in the classical case of non-causal state dependence without actions [8], in the present setting too feedback does not increase capacity.

\section{Causally Available States}

Consider now a setting similar to that of the previous section, with an action sequence, as before, of the form $A^{n}(M)$, but with a channel input restricted to causal dependence on the state sequence, i.e., of the form $X_{i}\left(M, S^{i}\right), 1 \leq i \leq n$. So the joint PMF of $M, A^{n}, S^{n}, X^{n}, Y^{n}$, induced by a given scheme, is

$$
P_{M, A^{n}, S^{n}, X^{n}, Y^{n}}\left(m, a^{n}, s^{n}, x^{n}, y^{n}\right)=\frac{1_{\left\{A^{n}(m)=a^{n}\right\}}}{|\mathcal{M}|} \prod_{i=1}^{n} P_{S \mid A}\left(s_{i} \mid a_{i}\right) 1_{\left\{X_{i}\left(m, s^{i}\right)=x_{i}\right\}} P_{Y \mid X, S}\left(y_{i} \mid x_{i}, s_{i}\right) .
$$

The associated probability of error is $P_{e}=P\left(M \neq \hat{M}\left(Y^{n}\right)\right)$, where $\hat{M}\left(Y^{n}\right)$ is the best (maximum likelihood) estimate of $M$ based on $Y^{n}$ under the joint distribution in (14). As before, the rate $R$ is said to be achievable if there exists a sequence of schemes for increasing block lengths with $\frac{1}{n} \log |\mathcal{M}| \leq R$ and $P_{e} \stackrel{n \rightarrow \infty}{\longrightarrow} 0$. The capacity of the channel with action-dependent states known causally to the decoder is the supremum over all achievable rates.

Theorem 2 The capacity of the channel with action-dependent states known causally to the decoder is given by

$$
C=\max I(U ; Y)
$$

where $U, A, S, X, Y$ are distributed according to

$$
P_{U, A, S, X, Y}(u, a, s, x, y)=P_{U}(u) 1_{\{g(u)=a\}} P_{S \mid A}(s \mid a) 1_{\{f(u, s)=x\}} P_{Y \mid X, S}(y \mid x, s),
$$

for some $P_{U}, f, g$ and $|\mathcal{U}| \leq \min \{|\mathcal{Y}|,|\mathcal{A}||\mathcal{S}| \mathcal{X} \mid+1\}$.

Comments: 
- Note the convexity of $I(U ; Y)$ in $P_{A \mid U}$ (due to its convexity in $P_{Y \mid U}$ ), which implies that the maximum in (15) would be unaffected when allowing a general $P_{A \mid U}$ rather than $A$ which is a function of $U$, as is implied by (16). Also, $A$ being a function of $U$ implies that the above maximization would remain unchanged when allowing an $f$ of the form $f(u, s, a)$. Since the convexity of $I(U ; Y)$ in $P_{A \mid U}$ also implies its convexity in $P_{X \mid U, S, A}$ for fixed $P_{U, S, A}$, it further follows that the maximum would be unaffected upon allowing a general $P_{X \mid U, S, A}$ in lieu of the $X=f(U, S)$ relationship implied in (17). Thus, $C$ in (15) can also be expressed as $C=\max I(U ; Y)$, where $U, A, S, X, Y$ are distributed according to

$$
P_{U, A, S, X, Y}(u, a, s, x, y)=P_{U, A}(u, a) P_{S \mid A}(s \mid a) P_{X \mid U, S, A}(x \mid u, s, a) P_{Y \mid X, S}(y \mid x, s),
$$

for some distributions $P_{U, A}$ and $P_{X \mid U, S, A}$. It follows that, given jointly distributed $U_{i}, A_{i}, S_{i}, X_{i}, Y_{i}$, to check that $I\left(U_{i} ; Y_{i}\right) \leq \max I(U ; Y)$ one need only verify that: $S_{i}-A_{i}-U_{i}$, that $P_{S_{i} \mid A_{i}}=P_{S \mid A}$, that $Y_{i}-\left(X_{i}, S_{i}\right)-\left(A_{i}, U_{i}\right)$, and that $P_{Y_{i} \mid X_{i}, S_{i}}=P_{Y \mid X, S}$. We will use this fact in the proof of Theorem 2.

- Since $I(U ; S \mid A)=0$ when $U-A-S$, the capacity expression for the causal case can be viewed as a maximization of the same functional as that for the non-causal case, but over a smaller set of distributions restricted to satisfy, in addition to the constraints from the non-causal case, the Markov relation $U-A-S$.

- Letting $C_{S}\left(P_{S}, P_{Y \mid X, S}\right)$ denote the capacity of the channel with states known causally at the transmitter, as considered in [11] (the subscript standing for 'Shannon'), a comment analogous to that made in the setting of non-causally available states, about the way that $\max _{a \in \mathcal{A}} C_{S}\left(P_{S \mid A=a}, P_{Y \mid X, S}\right)$ compares with the capacity characterized in Theorem 2, is applicable here.

Proof of Theorem 2: The achievability part follows similarly as in the original setting of [11], by constructing a good code for the standard problem of channel coding over the DMC from $U$ to $Y$. For the converse part, fix an arbitrary scheme and consider

$$
\begin{aligned}
I\left(M ; Y^{n}\right) & =H\left(Y^{n}\right)-H\left(Y^{n} \mid M\right) \\
& \leq \sum_{i=1}^{n} H\left(Y_{i}\right)-H\left(Y_{i} \mid M, Y^{i-1}\right) \\
& \leq \sum_{i=1}^{n} H\left(Y_{i}\right)-H\left(Y_{i} \mid M, Y^{i-1}, S^{i-1}\right) \\
& =\sum_{i=1}^{n} I\left(U_{i} ; Y_{i}\right) \\
& \leq n C,
\end{aligned}
$$

where the equality before last follows from defining $U_{i}=\left(M, Y^{i-1}, S^{i-1}\right)$. To see why the last inequality holds note the relations $S_{i}-A_{i}-U_{i}, P_{S_{i} \mid A_{i}}=P_{S \mid A}, Y_{i}-\left(X_{i}, S_{i}\right)-\left(U_{i}, A_{i}\right)$, and $P_{Y_{i} \mid X_{i}, S_{i}}=P_{Y \mid X, S}$, so it remains only to justify the bound on the cardinality of $|\mathcal{U}|$. That this cardinality need not be larger than that of the channel output alphabet follows from an argument identical to that given in [10] for why in the classical channel coding problem there is a capacity achieving distribution putting positive mass on a number of channel input symbols that is no larger than the number of channel output symbols. That it need not exceed $|\mathcal{A}||\mathcal{S}||\mathcal{X}|+1$ is due to an argument similar to that given at the end of the proof of Theorem 1: the requirement to preserve $H(S \mid U, A)$ is replaced by the requirement to preserve the Markov relation $S-A-U$ so the overall cardinality bound remains unchanged. The proof is completed via a standard use of Fano's inequality. 
It is readily checked that all the Markov and marginal distribution relations verified in the proof continue to hold when $A_{i}$ is allowed to depend on past states, i.e., to be of the form $A_{i}\left(M, S^{i-1}\right)$ rather than just $A_{i}(M)$. Thus, unlike for the setting of the previous section, here we have proof that 'feedback' from the past states at the action stage does not increase the capacity. In fact, said relations are readily verified to continue to hold for even more general action strategies where, in addition to the past channel states, there is feedback from the past channel outputs available, i.e., schemes of the form $A_{i}\left(M, S^{i-1}, Y^{i-1}\right){ }^{3}$ Finally, similarly as in the setting of non-causal encoding of the previous section, and as in the classical case of causal state dependence without actions [8], here too it can be seen that feedback at the encoding stage does not increase the capacity by verifying that the above relations continue to hold for channel inputs of the form $X_{i}\left(M, S^{i}, Y^{i-1}\right)$.

\section{Extensions, Equivalent Representations, and Special Cases}

\section{4-A Cost Constraints}

Similarly as in the classical problems, where it is often natural to introduce cost constraints on the channel input sequence, in our present setting it is natural to consider constraints on the cost of actions, of channel inputs, and of combinations thereof. Indeed, the cost in our setting, in its most general form, should be a function jointly of both the action and the channel input symbol. For example, when both actions and channel input symbols are real-valued, it is natural to constrain the power of the sum of those symbols, i.e., to consider the cost function $(a+x)^{2}$. Further, as in classical problems where one may be concerned say with both peak and power constraints, it will make sense to accommodate the possibility of $d \geq 1$ cost functions. Equivalently, we may assume one cost function of the form $\Lambda: \mathcal{A} \times \mathcal{X} \rightarrow \mathbb{R}^{d}$ and refer to

$$
E\left[\frac{1}{n} \sum_{i=1}^{n} \Lambda\left(A_{i}, X_{i}\right)\right]
$$

as the cost (vector) associated with a coding scheme. Given a vector $\lambda \in \mathbb{R}^{d}$, we refer to a rate $R$ as achievable at cost $\lambda$ if there exists a sequence of schemes for increasing block lengths with $\frac{1}{n} \log |\mathcal{M}| \leq R, P_{e} \stackrel{n \rightarrow \infty}{\longrightarrow} 0$, and $\limsup _{n \rightarrow \infty} E\left[\frac{1}{n} \sum_{i=1}^{n} \Lambda_{k}\left(A_{i}, X_{i}\right)\right] \leq \lambda_{k}$ for $1 \leq k \leq d$ (where $\Lambda_{k}$ and $\lambda_{k}$ denote the $k$ th coordinates of $\Lambda$ and $\lambda$ ). The capacity $C(\lambda)$ is the supremum over all rates achievable at cost $\lambda$.

Theorem 3 The capacities of the channel with action-dependent states known non-causally and causally to the transmitter, under a cost constraint $\lambda$, are given by the respective maximizations in Theorem 1 and Theorem 2, with the same cardinality bounds and an additional cost constraint

$$
E[\Lambda(A, X)] \leq \lambda
$$

Note that both sides of inequality (24) are $d$-dimensional vectors, and inequality between vectors is to be understood componentwise.

Proof of Theorem 3: We prove the Theorem for the case where states are known non-causally. Proof for the case of causally available states is similar (and simpler). That the introduction of cost constraints entails no increase in the cardinality of $\mathcal{U}$ follows from the fact that the preservation of $P_{A, S, X}$, which was argued in the absence of a cost constraint, automatically implies the preservation of $E[\Lambda(A, X)]$. Let now $C^{(I)}(\lambda)$ denote the maximum specified in Theorem 1, with the additional constraint $E[\Lambda(A, X)] \leq \lambda$. That $C(\lambda) \geq C^{(I)}(\lambda)$ follows from essentially the same

\footnotetext{
${ }^{3}$ Note that it is meaningless to consider schemes of this form in the setting of the previous section where the channel inputs (and hence outputs) are formed only after the whole state (and hence action) sequence has been formed.
} 
achievability arguments as in the case without a cost constraint. The converse, namely that $C(\lambda) \leq C^{(I)}(\lambda)$, follows similarly as in the unconstrained case once concavity of $C^{(I)}(\lambda)$ is established. To this end, define

$$
C_{Q}^{(I)}(\lambda)=\max [I(U ; Y \mid Q)-I(U ; S \mid A, Q)]
$$

where the maximization is over all joint distributions of the form

$$
P_{Q, A, S, U, X, Y}(q, a, s, u, x, y)=P_{Q}(q) P_{A \mid Q}(a \mid q) P_{S \mid A}(s \mid a) P_{U \mid S, A, Q}(u \mid s, a, q) 1_{\{x=f(u, q, s)\}} P_{Y \mid X, S}(y \mid x, s)
$$

for some $P_{Q}, P_{A \mid Q}, P_{U \mid S, A, Q}, f$ and such that $E[\Lambda(A, X)] \leq \lambda$. Thus, $C_{Q}^{(I)}(\lambda)$ is the 'concavification' of $C^{(I)}(\lambda)$ via the 'time-sharing' random variable $Q$. Since the maximum defining $C_{Q}^{(I)}(\lambda)$ is over a larger set than that in the definition of $C^{(I)}(\lambda)$, we obviously have $C_{Q}^{(I)}(\lambda) \geq C^{(I)}(\lambda)$. It remains to argue why $C_{Q}^{(I)}(\lambda) \leq C^{(I)}(\lambda)$. To this end note that under any distribution of the form in (26),

$$
\begin{aligned}
I(U ; Y \mid Q)-I(U ; S \mid A, Q) & =H(Y \mid Q)-H(Y \mid Q, U)-H(S \mid A, Q)-H(S \mid A, Q, U) \\
& \leq H(Y)-H(Y \mid Q, U)-H(S \mid A)-H(S \mid A, Q, U) \\
& =I(Q, U, Y)-I(Q, U ; S \mid A) \\
& =I\left(U^{\prime}, Y\right)-I\left(U^{\prime} ; S \mid A\right)
\end{aligned}
$$

where the inequality follows since $H(Y \mid Q) \leq H(Y)$ and $H(S \mid A, Q)=H(S \mid A)$ due to the Markov relation $S-A-Q$, and the last equality follows by letting $U^{\prime}=(Q, U)$. The proof is completed by noting that the joint distribution of $\left(A, S, U^{\prime}, X, Y\right)$ is of the form in the feasible set for the maximization defining $C^{(I)}(\lambda)$.

\section{4-B Equivalent Representations}

The capacity expression can equivalently be considered a maximization of $I(A, U ; Y)-I(U ; S \mid A)$, rather than $I(U ; Y)-I(U ; S \mid A)$. That the former is at least as large as the latter is clear. To see why the reverse inequality holds, note that $I(A, U ; Y)-I(U ; S \mid A)=I(A, U ; Y)-I(A, U ; S \mid A)=I\left(U^{\prime} ; Y\right)-I\left(U^{\prime} ; S \mid A\right)$, where $U^{\prime}=(U, A)$ and the joint distribution $\left(A, S, U^{\prime}, X, Y\right)$ satisfies the same conditional independence relations as $(A, S, U, X, Y)$. In some of the subsequent examples we will use this equivalent form.

Also, it might sometimes be natural to consider channels of the form $P_{Y \mid X, S, A}$. The capacity expressions for this seemingly more general channel remain almost unchanged, the only difference being that $X$ need be taken of the form $X(U, S, A)$ rather than $X(U, S)$. This follows directly by defining a new state $S^{\prime}=(S, A)$ and applying the original characterizations. We use these equivalent representations in some of the examples below.

\section{4-C Special Cases}

\section{4-C.1 Common Message Capacity of MAC with States at One Transmitter}

A setting considered in [12] is that of communicating a common message over a memoryless state-dependent multiple access channel characterized by $P_{Y \mid S, X_{1}, X_{2}}$, where the state sequence is known (non-causally) to the second encoder, but unknown at the first encoder and at the receiver. This problem can be seen as a special case of our setting via the following associations:

- $A \rightarrow X_{1}$

- $P_{S \mid A} \rightarrow P_{S}$ 
- $X \rightarrow X_{2}$

- $P_{Y \mid S, A, X} \rightarrow P_{Y \mid S, X_{1}, X_{2}}$

Applying Theorem 1 to this case, keeping the comment about channels of the form $P_{Y \mid S, A, X}$ from Subsection 4-B in mind, and noting that $I\left(U ; S \mid X_{1}\right)=I\left(U, X_{1} ; S\right)$ when $X_{1}$ and $S$ are independent, we get that the capacity is given by

$$
\max \left[I(U ; Y)-I\left(U, X_{1} ; S\right)\right]
$$

under joint distributions of the form

$$
P_{X_{1}}\left(x_{1}\right) P_{S}(s) P_{U \mid S, X_{1}}\left(u \mid s, x_{1}\right) 1_{\left\{x_{2}=f\left(u, s, x_{1}\right)\right\}} P_{Y \mid S, X_{1}, X_{2}}\left(y \mid s, x_{1}, x_{2}\right),
$$

where the maximization is over $P_{X_{1}}, P_{U \mid S, X_{1}}$, and $f$. This recovers Corollary 2 of [12].

\section{4-C.2 Actions Seen by Decoder}

Non-Causal Knowledge of States at Transmitter: Consider the case where the decoder has access to the actions taken. Noting that this is a special case of our setting by taking the pair $(Y, A)$ as the new channel output, that $U-(X, S, A)-Y$ if and only if $U-(X, S, A)-(Y, A)$, and the identity $I(A, U ; Y, U)-I(U ; S \mid A)=H(A)+I(U ; Y \mid A)-$ $I(U ; S \mid A)$, we obtain that the capacity for this case is given by

$$
\max [H(A)+I(U ; Y \mid A)-I(U ; S \mid A)]
$$

where the maximization is over the same set of distributions as in Theorem 1 . This expression is quite intuitive: The amount of information per symbol that can be conveyed through the actions in the first stage is represented by the term $H(A)$. In the second stage, both encoder and decoder know the action sequence, so can condition on it and proceed with ordinary Gelfand-Pinsker coding on each subsequence associated with each action symbol, achieving a rate $I(U ; Y \mid A)-I(U ; S \mid A)$. The maximization is a search for the optimal tradeoff between the amount of information that can be conveyed by the actions, and the quality of the Gelfand-Pinsker channel that they induce.

Causal Knowledge of States at Transmitter: Consider now the case where the states are known causally to the transmitter. Noting the same things as above, and the identity $I(U ; Y, A)=H(A)+I(U ; Y \mid A)$, we obtain that the capacity for this case is given by

$$
\max [H(A)+I(U ; Y \mid A)]
$$

where the maximization is over the same set of distributions as in Theorem 2. Analogously as in the preceding case, here the expression has a similar interpretation of conveying information in the first part via the selection of actions and then proceeding with coding for the ordinary Shannon channel on each subsequence associated with each action symbol.

\section{Channels with a Rewrite Option}

The generic framework considered thus far can be specialized to various scenarios involving coding for channels with a 'rewrite' option. We now detail some of these scenarios. 


\section{5-A Noise-Free Feedback}

Consider a DMC characterized by $P_{Y \mid X}$. After using the channel once and observing its output with no additional noise, the encoder makes another pass where it may rewrite at whichever locations it chooses, and the channel output after a rewrite will be an independent realization of the same channel $P_{Y \mid X}$. What is the capacity of such a coding scenario?

This can be cast into our framework via the following associations:

- $\mathcal{A} \rightarrow \mathcal{X}$ (alphabet of the first channel input)

- $\mathcal{S} \rightarrow \mathcal{Y}^{(1)}=\mathcal{Y}$, (the superscript (1) pertaining to the channel output after the first pass)

- $\mathcal{X} \rightarrow \tilde{\mathcal{X}}=\{$ no rewrite $\} \times \mathcal{X}$ (channel input alphabet in second pass)

- $\mathcal{Y} \rightarrow \mathcal{Y}^{(2)}=\mathcal{Y}$ (the superscript (2) pertaining to the channel output after the second pass)

- $P_{S \mid A} \rightarrow P_{Y^{(1)} \mid X}$, where $P_{Y^{(1)} \mid X}=P_{Y \mid X}$

- $P_{Y \mid X, S, A} \rightarrow P_{Y^{(2)} \mid X, Y^{(1)}, \tilde{X}}$, where

$$
P_{Y^{(2)} \mid X, Y^{(1)}, \tilde{X}}\left(y^{(2)} \mid x, y^{(1)}, \tilde{x}\right)=P_{Y^{(2)} \mid Y^{(1)}, \tilde{X}}\left(y^{(2)} \mid y^{(1)}, \tilde{x}\right)=\left\{\begin{array}{cc}
\mathbf{1}_{\left\{y^{(1)}=y^{(2)}\right\}} & \text { if } \tilde{x}=\text { no rewrite } \\
P_{Y \mid X}\left(y^{(2)} \mid \tilde{x}\right) & \text { otherwise }
\end{array}\right.
$$

Applying Theorem 1, with the above associations, and using the equivalent form discussed in Subsection 4-B, we get that the capacity for the case where the rewrite operations in the second pass may depend non-causally on the channel output from the first pass is given by

$$
\max \left[I\left(X, U ; Y^{(2)}\right)-I\left(U ; Y^{(1)} \mid X\right)\right]
$$

where the maximization is over all joint distributions of the form

$$
P_{X, Y^{(1)}, U, \tilde{X}, Y^{(2)}}\left(x, y^{(1)}, u, \tilde{x}, y^{(2)}\right)=P_{X}(x) P_{Y \mid X}\left(y^{(1)} \mid x\right) P_{U \mid Y^{(1)}, X}\left(u \mid y^{(1)}, x\right) 1_{\left\{\tilde{x}=f\left(u, y^{(1)}, x\right)\right\}} P_{Y^{(2)} \mid Y^{(1)}, \tilde{X}}\left(y^{(2)} \mid y^{(1)}, \tilde{x}\right),
$$

with $P_{Y^{(2)} \mid Y^{(1)}, \tilde{X}}$ given in (35), and where the maximization is over $P_{X}, P_{U \mid Y^{(1)}, X}, f$, with $|\mathcal{U}| \leq|\mathcal{X}||\mathcal{Y}|(|\mathcal{X}|+1)+1$.

Applying Theorem 2, with the above associations, we get that the capacity for the case where the rewrite operations in the second pass depend causally on the channel output components from the first pass is given by

$$
\max I\left(U ; Y^{(2)}\right)
$$

where $U, X, Y^{(1)}, \tilde{X}, Y^{(2)}$ are distributed according to

$$
P_{U, X, Y^{(1)}, \tilde{X}, Y^{(2)}}\left(u, x, y^{(1)}, \tilde{x}, y^{(2)}\right)=P_{U}(u) 1_{\{g(u)=x\}} P_{Y \mid X}\left(y^{(1)} \mid x\right) 1_{\left\{f\left(u, y^{(1)}\right)=\tilde{x}\right\}} P_{Y^{(2)} \mid Y^{(1)}, \tilde{X}}\left(y^{(2)} \mid y^{(1)}, \tilde{x}\right),
$$

with $P_{Y^{(2)} \mid Y^{(1)}, \tilde{X}}$ given in (35), and where the maximization is over $P_{U}, g, f$, with $|\mathcal{U}| \leq|\mathcal{Y}|$. In other words, perhaps not surprisingly given [11], capacity is achieved by coding for a memoryless channel whose input alphabet is $\mathcal{X} \times\{f: \mathcal{Y} \rightarrow \tilde{\mathcal{X}}\}$, output alphabet is $\mathcal{Y}$, and where the probability of a channel output symbol $y$ given input 'symbol' $(x, f(\cdot))$ is the probability that the symbol $y$ is eventually observed at the output of the channel when the symbol $x$ is first input into it and then $f(\cdot)$, evaluated at the output symbol in response to the first input, is used to determine whether and what will be inserted for the rewrite. 


\section{Example: BSC}

Consider the case where the channel is a $\operatorname{BSC}(\delta), 0 \leq \delta \leq 1 / 2$. Under any joint distribution allowed in the maximization in (38):

$$
\begin{aligned}
I\left(U ; Y^{(2)}\right) & =H\left(Y^{(2)}\right)-H\left(Y^{(2)} \mid U\right) \\
& \leq \log |\mathcal{Y}|-H\left(Y^{(2)} \mid U\right) \\
& \leq 1-h_{2}\left(\delta^{2}\right) .
\end{aligned}
$$

To see why the last inequality holds note that for all $u \in \mathcal{U}, H\left(Y^{(2)} \mid U=u\right)$ is the entropy of the channel output after the (option of a) rewrite operation when the first input is some deterministic symbol (which equals $g(u)$ ) and the rewrite operation is determined based on the channel output according to some deterministic function (which equals $f(u, \cdot)$ ). In the case of the BSC, this entropy is lower bounded by $h_{2}\left(\delta^{2}\right), \delta^{2}$ being the probability that a flip would occur twice in two consecutive uses of the channel. That $1-h_{2}\left(\delta^{2}\right)$ is indeed the capacity for this case follows from the fact that equality can be achieved in (41) and in (42) by taking in (39) $U \sim \operatorname{Bernoulli}(1 / 2), g$ the identity mapping, and $f$ to be given by

$$
f\left(u, y^{(1)}\right)=\left\{\begin{array}{cl}
\text { no rewrite } & \text { if } u=y^{(1)} \\
u & \text { otherwise. }
\end{array}\right.
$$

Moving to the case where the rewrite operations may depend non-causally on the channel output from the first pass, consider a joint distribution of the type allowed in (37), as follows: $X \sim \operatorname{Bernoulli}(1 / 2), Y^{(1)}$ is the output of $P_{Y \mid X}$ (the $\operatorname{BSC}(\delta)$ in this case), now generate $U \in\{0,1\}$ according to

$$
U=\left\{\begin{array}{cc}
0 & \text { if } Y^{(1)}=X \\
\operatorname{Bernoulli}(\alpha) & \text { otherwise }
\end{array}\right.
$$

where $0 \leq \alpha \leq 1$ is a parameter. Now let

$$
\tilde{X}=f\left(U, Y^{(1)}, X\right)=\left\{\begin{array}{cc}
\text { no rewrite } & \text { if } Y^{(1)}=X \text { or } U=1 \\
X & \text { otherwise }
\end{array}\right.
$$

and $Y^{(2)}$ is the output of the rewrite channel $P_{Y^{(2)} \mid Y^{(1)}, \tilde{X}}$. Under this joint distribution, simple computations give:

$$
\begin{gathered}
H\left(Y^{(2)}\right)=1, \\
H\left(Y^{(1)} \mid X\right)=h_{2}(\delta), \\
H\left(Y^{(1)} \mid X, U\right)=h_{2}\left(\delta \frac{1-\alpha}{1-\delta \alpha}\right)(1-\delta \alpha), \\
H\left(Y^{(2)} \mid X, U\right)=h_{2}\left(\delta^{2} \frac{1-\alpha}{1-\delta \alpha}\right)(1-\delta \alpha)
\end{gathered}
$$

so that

$$
\begin{aligned}
I\left(X, U ; Y^{(2)}\right)-I\left(U ; Y^{(1)} \mid X\right) & =H\left(Y^{(2)}\right)-H\left(Y^{(2)} \mid X, U\right)-H\left(Y^{(1)} \mid X\right)+H\left(Y^{(1)} \mid X, U\right) \\
& =1-h_{2}(\delta)+\left[h_{2}\left(\delta \frac{1-\alpha}{1-\delta \alpha}\right)-h_{2}\left(\delta^{2} \frac{1-\alpha}{1-\delta \alpha}\right)\right](1-\delta \alpha) .
\end{aligned}
$$

As is to be expected, when $\alpha=0, U$ is degenerate, so we recover a joint distribution of the type allowed and achieving the maximum in the causal case, and indeed the expression in (48) becomes $1-h_{2}\left(\delta^{2}\right)$ when $\alpha=0$. However, we may now optimize over $\alpha$ to obtain the following lower bound on the capacity of the BSC with a rewrite option for the non-causal case:

$$
1-h_{2}(\delta)+\max _{0 \leq \alpha \leq 1}\left\{\left[h_{2}\left(\delta \frac{1-\alpha}{1-\delta \alpha}\right)-h_{2}\left(\delta^{2} \frac{1-\alpha}{1-\delta \alpha}\right)\right](1-\delta \alpha)\right\}
$$


To see that this capacity can be strictly higher than its counterpart for the causality-constrained scenario, consider the case $\delta=1 / 2$. A straightforward calculation shows that in this case (49) assumes the value $\approx 0.207519(\alpha=1 / 3$ achieves the maximum), as compared to $1-h_{2}(1 / 4) \approx 0.188722$, which is the capacity under the causality constraint. Thus, for the $\mathrm{BSC}(1 / 2)$, relaxation of the causality constraint boosts the rewrite capacity by at least $10 \%$.

\section{5-B Channels with a Rewrite Option based on Noisy Feedback}

We now generalize the setting of the previous subsection to the more realistic scenario where the rewrite decision is based on a noisy observation of the channel outputs from the first pass. The forward channel is, as before, a DMC

$P_{Y \mid X}$, while the channel outputs from the first pass are observed by the rewrite encoder through a DMC $P_{Z \mid Y}$, the 'backward' channel. What is the rewrite capacity in this noisy setting?

This can be cast into our framework via the following associations:

- $\mathcal{A} \rightarrow \mathcal{X}$ (alphabet of the first channel input)

- $\mathcal{S} \rightarrow \mathcal{Z}$ (alphabet of noisy observation of the first channel input)

- $\mathcal{X} \rightarrow \tilde{\mathcal{X}}=\{$ no rewrite $\} \times \mathcal{X}$ (channel input alphabet in second pass)

- $\mathcal{Y} \rightarrow \mathcal{Y}^{(2)}=\mathcal{Y}$ (the superscript (2) pertaining to the channel output after the second pass)

- $P_{S \mid A} \rightarrow P_{Z \mid X}$, where $P_{Z \mid X}(z \mid x)=\sum_{y} P_{Y \mid X}(y \mid x) P_{Z \mid Y}(z \mid y)$

- $P_{Y \mid X, S, A} \rightarrow P_{Y^{(2)} \mid \tilde{X}, Z, X}$ which is explicitly given by

$$
P_{Y^{(2)} \mid \tilde{X}, Z, X}\left(y^{(2)} \mid \tilde{x}, z, x\right)=\left\{\begin{array}{cc}
P_{Y \mid X, Z}\left(y^{(2)} \mid x, z\right) & \text { if } \tilde{x}=\text { no rewrite } \\
P_{Y \mid X}\left(y^{(2)} \mid \tilde{x}\right) & \text { otherwise }
\end{array}\right.
$$

where $P_{Y \mid X, Z}$ is induced by $P_{Y \mid X}$ and $P_{Z \mid Y}$, namely

$$
P_{Y \mid X, Z}(y \mid x, z)=\frac{P_{Y \mid X}(y \mid x) P_{Z \mid Y}(z \mid y)}{\sum_{y^{\prime}} P_{Y \mid X}\left(y^{\prime} \mid x\right) P_{Z \mid Y}\left(z \mid y^{\prime}\right)} .
$$

Applying Theorem 2, with the above associations, we get that the capacity for the case where the rewrite operations in the second pass depend causally on the channel output components from the first pass is given by

$$
\max I\left(U ; Y^{(2)}\right),
$$

where $U, X, Y^{(1)}, Z, \tilde{X}, Y^{(2)}$ are distributed according to

$$
P_{U, X, Y^{(1)}, Z, \tilde{X}, Y^{(2)}}\left(u, x, y^{(1)}, z, \tilde{x}, y^{(2)}\right)=P_{U}(u) 1_{\{g(u)=x\}} P_{Y \mid X}\left(y^{(1)} \mid x\right) P_{Z \mid Y}\left(z \mid y^{(1)}\right) 1_{\{f(u, z)=\tilde{x}\}} P_{Y^{(2)} \mid \tilde{X}, Z, X}\left(y^{(2)} \mid \tilde{x}, z, x\right),
$$

with $P_{Y^{(2)} \mid \tilde{X}, Z, X}$ given in (50), and where the maximization is over $P_{U}, g, f$, with $|\mathcal{U}| \leq \min \{|\mathcal{Y}|,|\mathcal{X}|(|\mathcal{X}|+1)|\mathcal{Z}|+1\}$. In other words, capacity is achieved by coding for a memoryless channel whose input alphabet is $\mathcal{X} \times\{f: \mathcal{Y} \rightarrow \tilde{\mathcal{X}}\}$, output alphabet is $\mathcal{Y}$, and where the probability of a channel output symbol $y$ given input 'symbol' $(x, f(\cdot))$ is the probability that the symbol $y$ is eventually observed at the output of the channel when the symbol $x$ is first input into it and then $f(\cdot)$, evaluated at the noisy measurement of the channel output after the first input, is used to determine whether that location will be rewritten to and, if so, what will the new input symbol be. 


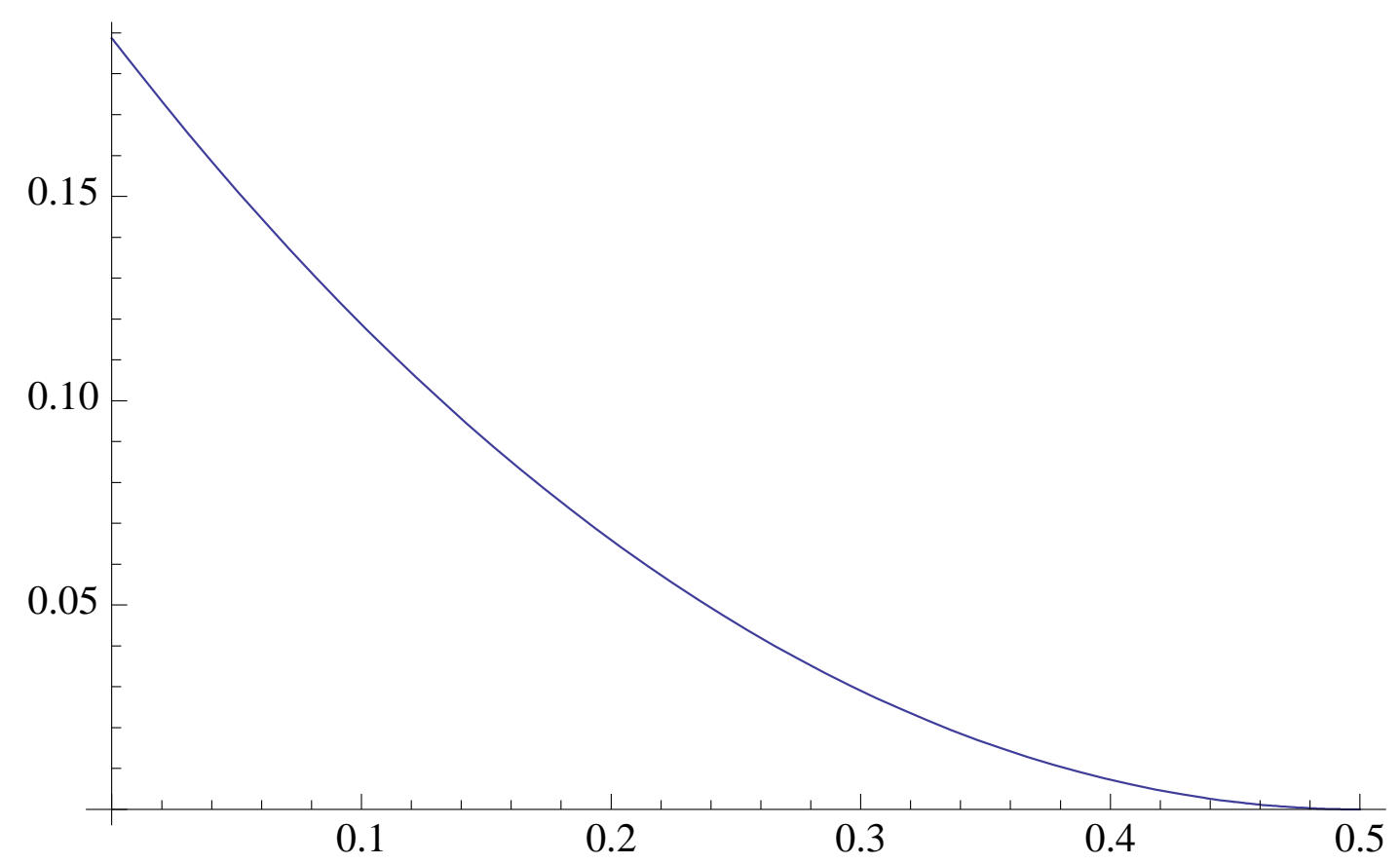

Figure 2: Capacity of $\mathrm{BSC}(\delta)$ with rewrite based on $\mathrm{BSC}(\varepsilon)$-corrupted feedback from the first pass, when the rewrite is restricted to causal dependence on the channel outputs from the first pass. Plotted here for $\delta=1 / 2$, as a function of $\varepsilon$.

Applying Theorem 1, with the above associations, we get that the capacity for the case where the rewrite operations in the second pass may depend non-causally on the channel output from the first pass is given by

$$
\max \left[I\left(X, U ; Y^{(2)}\right)-I(U ; Z \mid X)\right]
$$

where the maximization is over all joint distributions of the form

$P_{X, Y^{(1)}, Z, U, \tilde{X}, Y^{(2)}}\left(x, y^{(1)}, u, \tilde{x}, y^{(2)}\right)=P_{X}(x) P_{Y \mid X}\left(y^{(1)} \mid x\right) P_{Z \mid Y}\left(z \mid y^{(1)}\right) P_{U \mid Z, X}(u \mid z, x) 1_{\{\tilde{x}=f(u, z, x)\}} P_{Y^{(2)} \mid \tilde{X}, Z, X}\left(y^{(2)} \mid \tilde{x}, z, x\right)$,

with $P_{Y^{(2)} \mid \tilde{X}, Z, X}$ given in (50), and where the maximization is over $P_{X}, P_{U \mid Z, X}, f$, with $|\mathcal{U}| \leq|\mathcal{X}|(|\mathcal{X}|+1)|\mathcal{Z}|+1$.

\section{Example: BSC}

Consider the case where both the forward and the backward channels are BSCs with respective parameters $0 \leq \delta \leq$ $1 / 2$ and $0 \leq \varepsilon \leq 1 / 2$. For the case where the rewrite operations in the second pass depend causally on the (noisy) observations of the channel output components from the first pass, the arguments in Subsection 5-A carry over to this noisy scenario and imply that the capacity achieving triple $(U, g, f)$ for the causal case is the same as that for the noise-free backward channel. Under this triple, a simple calculation shows that the channel from $X$ to $Y^{(2)}$ is a BSC with crossover probability $\varepsilon \delta(2-\delta)+\delta^{2}(1-\varepsilon)$, so the resulting capacity is

$$
1-h_{2}\left(\varepsilon \delta(2-\delta)+\delta^{2}(1-\varepsilon)\right)
$$

which is plotted in Figure 2. Note, in particular, the two extremes: when $\varepsilon=0$, we recover the $1-h_{2}\left(\delta^{2}\right)$ of Subsection 5 -A while for $\varepsilon=1 / 2,(56)$ becomes $1-h_{2}(\delta)$, capacity of the vanilla $\operatorname{BSC}(\delta)$. 
Moving to the case where the rewrite operations may depend non-causally on the channel output from the first pass, consider a joint distribution of the type allowed in (55), as follows: $X \sim \operatorname{Bernoulli}(1 / 2), Y^{(1)}$ is the output of $P_{Y \mid X}$ (the $\operatorname{BSC}(\delta)$ in this case), $Z$ is the output of $P_{Z \mid Y^{(1)}}$ (the $\operatorname{BSC}(\varepsilon)$ in this case), now generate $U \in\{0,1\}$ according to

$$
U=\left\{\begin{array}{cc}
0 & \text { if } Z=X \\
\operatorname{Bernoulli}(\alpha) & \text { otherwise }
\end{array}\right.
$$

where $0 \leq \alpha \leq 1$ is a parameter. Let now

$$
\tilde{X}=f(U, Z, X)=\left\{\begin{array}{cc}
\text { no rewrite } & \text { if } Z=X \text { or } U=1 \\
X & \text { otherwise }
\end{array}\right.
$$

and $Y^{(2)}$ is the output of the rewrite channel $P_{Y^{(2)} \mid \tilde{X}, Z, X}$ in (50) which in this case is given by

$$
P_{Y^{(2)} \mid \tilde{X}, Z, X}\left(y^{(2)} \mid \tilde{x}, z, x\right)=\left\{\begin{array}{cc}
P_{Y \mid X, Z}\left(y^{(2)} \mid x, z\right) & \text { if } \tilde{x}=\text { no rewrite } \\
1_{\left\{y^{(2)}=\tilde{x}\right\}}(1-\delta)+1_{\left\{y^{(2)} \neq \tilde{x}\right\}} \delta & \text { otherwise }
\end{array}\right.
$$

where

$$
P_{Y \mid X, Z}(y \mid x, z)=\left\{\begin{array}{cl}
\frac{(1-\delta)(1-\varepsilon)}{1-\delta * \varepsilon} & \text { if }(y, x, z)=(0,0,0) \text { or }(y, x, z)=(1,1,1) \\
1-\frac{(1-\delta)(1-\varepsilon)}{1-\delta \varepsilon} & \text { if }(y, x, z)=(1,0,0) \text { or }(y, x, z)=(0,1,1) \\
\frac{(1-\delta) \varepsilon}{\delta * \varepsilon} & \text { if }(y, x, z)=(0,0,1) \text { or }(y, x, z)=(1,1,0) \\
1-\frac{(1-\delta) \varepsilon}{\delta * \varepsilon} & \text { if }(y, x, z)=(1,0,1) \text { or }(y, x, z)=(0,1,0) .
\end{array}\right.
$$

and $*$ denotes binary convolution defined by $\varepsilon * \delta=\varepsilon(1-\delta)+\delta(1-\varepsilon)$. The above mappings and conditional distributions further induce the following joint and conditional PMFs:

$$
\begin{aligned}
& P_{X, Z, U}(x, z, u)=\left\{\begin{array}{cl}
\frac{1}{2}(1-\delta * \varepsilon) & \text { if }(x, z, u)=(0,0,0) \text { or }(x, z, u)=(1,1,0) \\
\frac{1}{2}(\delta * \varepsilon)(1-\alpha) & \text { if }(x, z, u)=(0,1,0) \text { or }(x, z, u)=(1,0,0) \\
\frac{1}{2}(\delta * \varepsilon) \alpha & \text { if }(x, z, u)=(0,1,1) \text { or }(x, z, u)=(1,0,1) \\
0 & \text { if }(x, z, u)=(0,0,1) \text { or }(x, z, u)=(1,1,1)
\end{array}\right. \\
& P_{Y^{(2)} \mid X, U}\left(y^{(2)} \mid x, u\right) \\
& =\sum_{z} P_{Y^{(2)}, Z \mid X, U}\left(y^{(2)}, z \mid x, u\right) \\
& =\sum_{z} P_{Z \mid X, U}(z \mid x, u) P_{Y^{(2)} \mid U, Z, X}\left(y^{(2)} \mid u, z, x\right) \\
& =\sum_{z} P_{Z \mid X, U}(z \mid x, u) P_{Y^{(2)} \mid \tilde{X}, Z, X}\left(y^{(2)} \mid f(u, z, x), z, x\right) \\
& =\left\{\begin{array}{cc}
\frac{(1-\delta * \varepsilon)}{(1-\delta * \varepsilon)+(\delta * \varepsilon)(1-\alpha)} \frac{(1-\delta)(1-\varepsilon)}{(1-\delta * \varepsilon)}+\frac{(\delta * \varepsilon)(1-\alpha)}{(1-\delta * \varepsilon)+(\delta * \varepsilon)(1-\alpha)}(1-\delta) & \text { if }\left(y^{(2)}, x, u\right)=(0,0,0) \\
\frac{(1-\delta) \varepsilon}{\delta * \varepsilon} & \text { if }\left(y^{(2)}, x, u\right)=(0,0,1) .
\end{array}\right.
\end{aligned}
$$

Equipped with the PMFs in (61) and (62), the entropies are readily obtained as:

$$
\begin{gathered}
H\left(Y^{(2)}\right)=1, \\
H(Z \mid X)=h_{2}(\delta * \varepsilon), \\
H(Z \mid X, U)=h_{2}\left(\frac{1-\delta * \varepsilon}{1-\delta * \varepsilon+(\delta * \varepsilon)(1-\alpha)}\right)[1-\delta * \varepsilon+(\delta * \varepsilon)(1-\alpha)], \\
H\left(Y^{(2)} \mid X, U\right)=h_{2}\left(\frac{(1-\delta)(1-\varepsilon)+(\delta * \varepsilon)(1-\alpha)(1-\delta)}{1-\delta * \varepsilon+(\delta * \varepsilon)(1-\alpha)}\right)[1-\delta * \varepsilon+(\delta * \varepsilon)(1-\alpha)]+h_{2}\left(\frac{(1-\delta) \varepsilon}{\delta * \varepsilon}\right)(\delta * \varepsilon) \alpha
\end{gathered}
$$


so that

$$
\begin{aligned}
& I\left(X, U ; Y^{(2)}\right)-I(U ; Z \mid X) \\
= & H\left(Y^{(2)}\right)-H\left(Y^{(2)} \mid X, U\right)-H(Z \mid X)+H(Z \mid X, U) \\
= & 1-h_{2}(\delta * \varepsilon) \\
& +\left[h_{2}\left(\frac{1-\delta * \varepsilon}{1-\delta * \varepsilon+(\delta * \varepsilon)(1-\alpha)}\right)-h_{2}\left(\frac{(1-\delta)(1-\varepsilon)+(\delta * \varepsilon)(1-\alpha)(1-\delta)}{1-\delta * \varepsilon+(\delta * \varepsilon)(1-\alpha)}\right)\right][1-\delta * \varepsilon+(\delta * \varepsilon)(1-\alpha)] \\
& -h_{2}\left(\frac{(1-\delta) \varepsilon}{\delta * \varepsilon}\right)(\delta * \varepsilon) \alpha . \\
= & 1-h_{2}(\delta * \varepsilon) \\
& +\left[h_{2}\left(\frac{1-\delta * \varepsilon}{1-(\delta * \varepsilon) \alpha}\right)-h_{2}\left(\frac{(1-\delta)(1-\varepsilon)+(\delta * \varepsilon)(1-\alpha)(1-\delta)}{1-(\delta * \varepsilon) \alpha}\right)\right][1-(\delta * \varepsilon) \alpha]-h_{2}\left(\frac{(1-\delta) \varepsilon}{\delta * \varepsilon}\right)(\delta * \varepsilon) \alpha .
\end{aligned}
$$

As would be expected, and as is easily verified, (64) coincides with (56) when $\alpha=0$ and with (48) when $\varepsilon=0$. Optimizing over $\alpha$, we obtain the following lower bound on the capacity of the BSC with a rewrite option based on a noisy observation of the channel output, for the non-causal case:

$$
\begin{aligned}
& 1-h_{2}(\delta * \varepsilon) \\
& +\max _{0 \leq \alpha \leq 1}\left\{\left[h_{2}\left(\frac{1-\delta * \varepsilon}{1-(\delta * \varepsilon) \alpha}\right)-h_{2}\left(\frac{(1-\delta)(1-\varepsilon)+(\delta * \varepsilon)(1-\alpha)(1-\delta)}{1-(\delta * \varepsilon) \alpha}\right)\right][1-(\delta * \varepsilon) \alpha]-h_{2}\left(\frac{(1-\delta) \varepsilon}{\delta * \varepsilon}\right)(\delta * \varepsilon) \alpha\right\} .
\end{aligned}
$$

Figure 3 presents a plot of the expression in (65), and one of the capacity when the rewrite is restricted to causality, when the forward channel is a $\operatorname{BSC}(1 / 2)$ and the backward one is $\operatorname{BSC}(\varepsilon)$.

\section{5-C Computer Memory with Defects and a Rewrite Option}

Consider a computer memory with defects, characterized by the distribution of the state of each cell, $P_{S}$, and the channel $P_{Y \mid X, S}$. Consider the following two-pass coding scenario: the memory state is known neither to the encoder nor the decoder. After writing into the storage device and observing the channel output components, the encoder makes another encoding pass where it may rewrite at whichever memory locations it chooses. At each memory location, the state remains unchanged regardless of whether or not a rewrite was performed. What is the storage capacity of such a two-pass coding device?

This can be cast into our framework via the following associations:

- $\mathcal{A} \rightarrow \mathcal{X}$

- $\mathcal{S} \rightarrow \mathcal{Y}^{(1)}=\mathcal{Y}$, (the superscript (1) pertaining to the channel output after the first pass)

- $\mathcal{X} \rightarrow \tilde{X}=\{$ no rewrite $\} \times \mathcal{X}$

- $\mathcal{Y} \rightarrow \mathcal{Y}^{(2)}=\mathcal{Y}$ (the superscript (2) pertaining to the channel output after the second pass)

- $P_{S \mid A} \rightarrow P_{Y^{(1)} \mid X}$, where $P_{Y^{(1)} \mid X}$ is the $P_{Y \mid X}$ induced by the original channel, i.e.,

$$
P_{Y^{(1)} \mid X}(y \mid x)=\sum_{s} P_{S}(s) P_{Y \mid X, S}(y \mid x, s)
$$




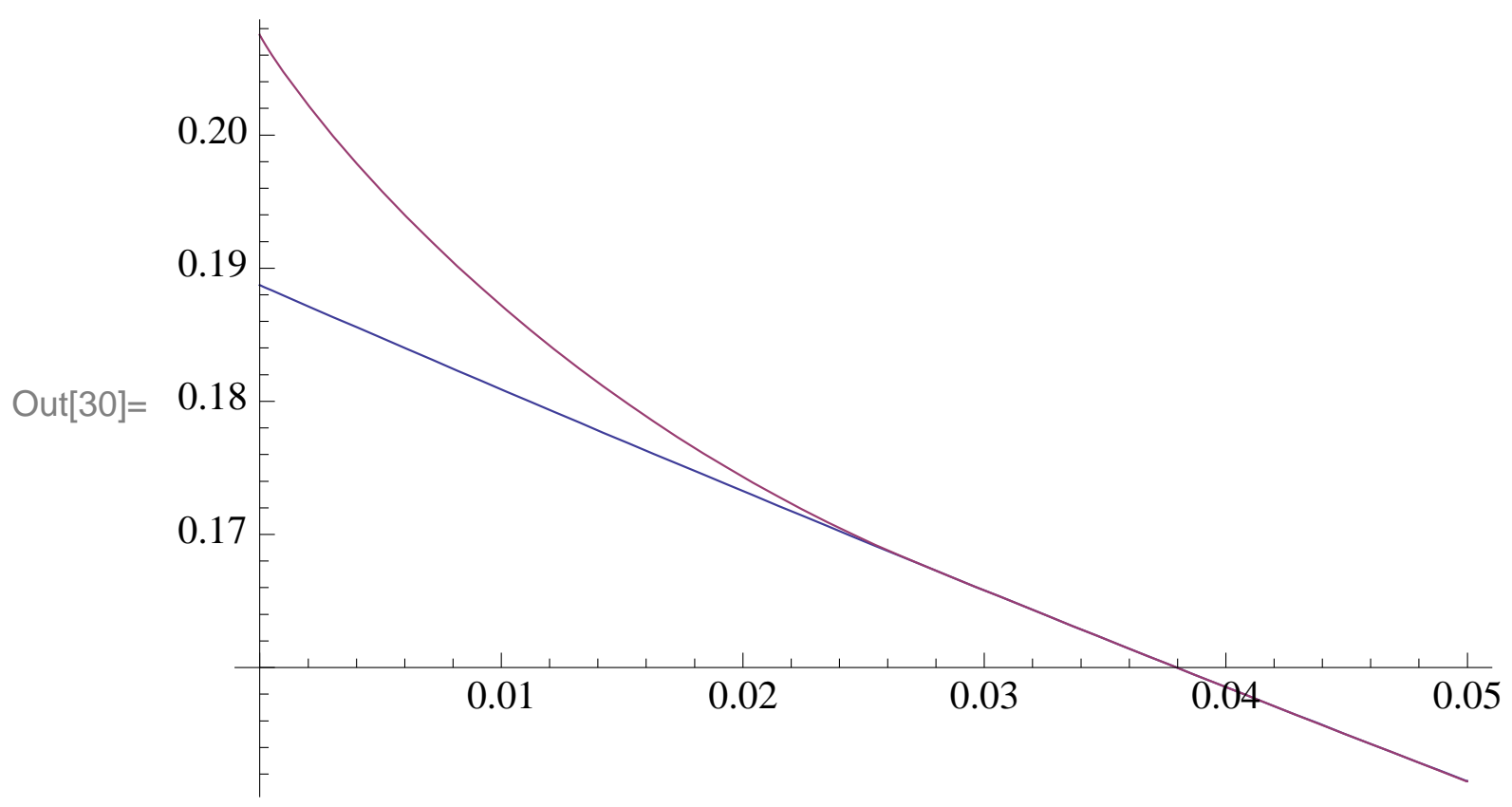

Figure 3: The lower curve is the actual capacity of a $\operatorname{BSC}(1 / 2)$ with a rewrite option based on observation of the channel outputs from the first pass through a $\mathrm{BSC}(\varepsilon)$, when the rewrite is restricted to causality, as given in (56). The upper curve is the lower bound on the capacity when there is no restriction to causality, as given in (65). The two curves touch the $Y$-axis at the points mentioned in Subsection 5 -A, namely $\approx 0.207519(\alpha=1 / 3$ achieves the maximum in (65) for this case), as compared to $1-h_{2}(1 / 4) \approx 0.188722$.

- $P_{Y \mid X, S, A} \rightarrow P_{Y^{(2)} \mid X, Y^{(1)}, \tilde{X}}$, where

$$
P_{Y^{(2)} \mid X, Y^{(1)}, \tilde{X}}\left(y^{(2)} \mid x, y^{(1)}, \tilde{x}\right)=\left\{\begin{array}{cc}
\mathbf{1}_{\left\{y^{(1)}=y^{(2)}\right\}} & \text { if } \tilde{x}=\text { no rewrite } \\
\sum_{s} P_{Y \mid X, S}\left(y^{(2)} \mid \tilde{x}, s\right) P_{S \mid X, Y}\left(s \mid x, y^{(1)}\right) & \text { otherwise, }
\end{array}\right.
$$

where $P_{S \mid X, Y}$ is the posterior distribution on the state given knowledge of the channel input and output, as induced by the original channel, namely

$$
P_{S \mid X, Y}(s \mid x, y)=\frac{P_{S, Y \mid X}(s, y \mid x)}{P_{Y \mid X}(y \mid x)}=\frac{P_{S}(s) P_{Y \mid X, S}(y \mid x, s)}{\sum_{s^{\prime}} P_{S}\left(s^{\prime}\right) P_{Y \mid X, S}\left(y \mid x, s^{\prime}\right)} .
$$

Note that $Y^{(1)}$ is affected by the encoding 'action' chosen for the first pass, and is then observed by the encoder before choosing its channel input symbol for the second pass. Further, knowledge of $Y^{(1)}$ conveys information about the state $S$, and thus affects the conditional distribution of the channel output if a rewrite operation is selected, so is playing the role of the channel state when cast into our general setting.

Applying Theorem 2, with the above associations, we get that the capacity for the case where the rewrite operations in the second pass depend causally on the channel output components from the first pass $C{ }_{C}^{C M D R W},{ }^{4}$ is given by

$$
C_{C}^{C M D R W}=\max I\left(U ; Y^{(2)}\right),
$$

where $U, X, Y^{(1)}, \tilde{X}, Y^{(2)}$ are distributed according to

$$
P_{U, X, Y^{(1)}, \tilde{X}, Y^{(2)}}\left(u, x, y^{(1)}, \tilde{x}, y^{(2)}\right)=P_{U}(u) 1_{\{g(u)=x\}} P_{Y^{(1)} \mid X}\left(y^{(1)} \mid x\right) 1_{\left\{f\left(u, y^{(1)}\right)=\tilde{x}\right\}} P_{Y^{(2)} \mid X, Y^{(1)}, \tilde{X}}\left(y^{(2)} \mid x, y^{(1)}, \tilde{x}\right),
$$

\footnotetext{
${ }^{4}$ The superscript in $C_{C}^{C M D R W}$ standing for 'Computer Memory with Defects and a ReWrite option' while the subscript stands for 'Causal'.
} 
with $P_{Y^{(1)} \mid X}$ and $P_{Y^{(2)} \mid X, Y^{(1)}, \tilde{X}}$ given in (66) and (67), and where the maximization is over $P_{U}, g$, f, with $|\mathcal{U}| \leq|\mathcal{Y}|$.

Applying Theorem 1, with the above associations, we get that the capacity for the case where the rewrite operations in the second pass may depend non-causally on the channel output components from the first pass $C_{N C}^{C M D R W},{ }^{5}$ is given by

$$
C_{N C}^{C M D R W}=\max \left[I\left(X, U ; Y^{(2)}\right)-I\left(U ; Y^{(1)} \mid X\right)\right]
$$

where the maximization is over all joint distributions of the form

$P_{X, Y^{(1)}, U, \tilde{X}, Y^{(2)}}\left(x, y^{(1)}, u, \tilde{x}, y^{(2)}\right)=P_{X}(x) P_{Y^{(1)} \mid X}\left(y^{(1)} \mid x\right) P_{U \mid Y^{(1)}, X}\left(u \mid y^{(1)}, x\right) 1_{\left\{\tilde{x}=f\left(u, y^{(1)}, x\right)\right\}} P_{Y^{(2)} \mid X, Y^{(1)}, \tilde{X}}\left(y^{(2)} \mid x, y^{(1)}, \tilde{x}\right)$,

with $P_{Y^{(1)} \mid X}$ and $P_{Y^{(2)} \mid X, Y^{(1)}, \tilde{X}}$ given in (66) and (67), and where the maximization is over $P_{X}, P_{U \mid Y^{(1)}, X}, f$, with $|\mathcal{U}| \leq|\mathcal{X}|(|\mathcal{X}|+1)|\mathcal{Y}|+1$.

\section{5-C.1 Example: A BSC that gets Stuck}

Consider a channel whose input-output relation can be in one of the following four states: $Y \equiv X, Y \equiv X \oplus 1, Y \equiv 0$, $Y \equiv 1$, with respective probabilities $(1-\delta)^{2}, \delta(1-\delta), \delta(1-\delta), \delta^{2}$. When the states are unkown, and there is no rewrite option, this is nothing but a $\operatorname{BSC}(\delta)$. Let us now compute the rewrite capacities, with and without the causality restriction, (69) and (71) for this channel. Note that a rewrite with the same symbol is pointless since would yield the same output. Thus, for this channel, if a rewrite operation is chosen, it can, without loss of optimality, always be taken to the value which is complementary to that used in the first pass. This fact simplifies the derivation.

It is readily verified that the maximum in (69) is achieved by the following encoding: if the symbol output by the channel in response to the first input is equal to that input, no rewrite is performed. Otherwise, a rewrite is performed with the complementary symbol. We obtain an effective BSC with crossover probability $\delta(1-\delta)$, and thus

$$
C_{C}^{C M D R W}=1-h_{2}(\delta(1-\delta))
$$

Moving to the evaluation of (71), symmetry implies that the most general form of a joint distribution we need to consider and optimize over is a follows: $P_{X}$ is $\operatorname{Bernoulli}(1 / 2), P_{Y^{(1)} \mid X}$ is the $\operatorname{BSC}(\delta), \mathcal{U}=\{0,1\}$ and $P_{U \mid Y^{(1)}, X}$ is of the form

$$
P_{U \mid Y^{(1)}, X}\left(1 \mid y^{(1)}, x\right)=\left\{\begin{array}{cc}
1-\alpha & \text { if } y^{(1)}=x=1 \\
\alpha & \text { if } y^{(1)}=x=0 \\
\beta & \text { if } y^{(1)} \neq x=0 \\
1-\beta & \text { if } y^{(1)} \neq x=1
\end{array}\right.
$$

Since, when a rewrite is performed, it need only be to the value which is complementary to that used in the first pass, there is no loss in assuming that $f$ is of the form

$$
f\left(u, y^{(1)}, x\right)=\left\{\begin{array}{cl}
\text { no rewrite } & \text { if }\left(u, y^{(1)}, x\right) \in\{(0,0,0),(1,1,1),(1,1,0),(0,0,1)\} \\
x \oplus 1 & \text { if }\left(u, y^{(1)}, x\right) \in\{(1,0,0),(0,1,1),(0,1,0),(1,0,1)\} .
\end{array}\right.
$$

Let us now compute the induced joint distributions:

$$
P_{Y^{(1)}, X, U}\left(y^{(1)}, x, u\right)=\left\{\begin{array}{cl}
\frac{1}{2}(1-\delta)(1-\alpha) & \text { if }\left(y^{(1)}, x, u\right) \in\{(0,0,0),(1,1,1)\} \\
\frac{1}{2}(1-\delta) \alpha & \text { if }\left(y^{(1)}, x, u\right) \in\{(0,0,1),(1,1,0)\} \\
\frac{1}{2} \delta \beta & \text { if }\left(y^{(1)}, x, u\right) \in\{(0,1,0),(1,0,1)\} \\
\frac{1}{2} \delta(1-\beta) & \text { if }\left(y^{(1)}, x, u\right) \in\{(0,1,1),(1,0,0)\},
\end{array}\right.
$$

\footnotetext{
${ }^{5}$ The subscript in $C_{N C}^{C M D R W}$ standing for 'Non-Causal'.
} 


$$
\begin{gathered}
P_{X, U}(x, u)=\left\{\begin{array}{cc}
\frac{1}{2}(1-\delta)(1-\alpha)+\frac{1}{2} \delta(1-\beta) & \text { if }(x, u)=(0,0) \\
\frac{1}{2}(1-\delta) \alpha+\frac{1}{2} \delta \beta & \text { if }(x, u)=(0,1) \\
\frac{1}{2} \delta \beta+\frac{1}{2}(1-\delta) \alpha & \text { if }(x, u)=(1,0) \\
\frac{1}{2} \delta(1-\beta)+\frac{1}{2}(1-\delta)(1-\alpha) & \text { if }(x, u)=(1,1)\},
\end{array}\right. \\
P_{Y^{(2)} \mid Y^{(1)}, X, U}\left(1 \mid y^{(1)}, x, u\right)=\left\{\begin{array}{cl}
0 & \text { if }\left(y^{(1)}, x, u\right) \in\{(0,0,0),(0,1,0)\} \\
1 & \text { if }\left(y^{(1)}, x, u\right) \in\{(1,1,1),(1,0,1)\} \\
\delta & \text { if }\left(y^{(1)}, x, u\right) \in\{(1,1,0),(0,1,1)\} \\
1-\delta & \text { if }\left(y^{(1)}, x, u\right) \in\{(0,0,1),(1,0,0)\},
\end{array}\right.
\end{gathered}
$$

$P_{Y^{(2)}, X, U}\left(y^{(2)}, x, u\right)=\sum_{y^{(1)}} P_{Y^{(2)}, Y^{(1)}, X, U}\left(y^{(2)}, y^{(1)}, x, u\right)=\left\{\begin{array}{cl}\frac{1}{2}(1-\delta)(1-\alpha)+\frac{1}{2} \delta^{2}(1-\beta) & \text { if }\left(y^{(2)}, x, u\right)=(0,0,0) \\ \frac{1}{2}(1-\delta) \alpha \delta & \text { if }\left(y^{(2)}, x, u\right)=(0,0,1) \\ \frac{1}{2} \delta \beta+\frac{1}{2}(1-\delta)^{2} \alpha & \text { if }\left(y^{(2)}, x, u\right)=(0,1,0) \\ \frac{1}{2}(1-\delta)(1-\beta) \delta & \text { if }\left(y^{(2)}, x, u\right)=(0,1,1) .\end{array}\right.$

So

$$
\begin{aligned}
& H\left(Y^{(1)} \mid X, U\right) \\
= & h_{2}\left(\frac{\frac{1}{2}(1-\delta)(1-\alpha)}{\frac{1}{2}(1-\delta)(1-\alpha)+\frac{1}{2} \delta(1-\beta)}\right)\left[\frac{1}{2}(1-\delta)(1-\alpha)+\frac{1}{2} \delta(1-\beta)\right] \\
& +h_{2}\left(\frac{\frac{1}{2}(1-\delta) \alpha}{\frac{1}{2}(1-\delta) \alpha+\frac{1}{2} \delta \beta}\right)\left[\frac{1}{2}(1-\delta) \alpha+\frac{1}{2} \delta \beta\right] \\
& +h_{2}\left(\frac{\frac{1}{2} \delta \beta}{\frac{1}{2} \delta \beta+\frac{1}{2}(1-\delta) \alpha}\right)\left[\frac{1}{2} \delta \beta+\frac{1}{2}(1-\delta) \alpha\right] \\
& +h_{2}\left(\frac{\frac{1}{2} \delta(1-\beta)}{\frac{1}{2} \delta(1-\beta)+\frac{1}{2}(1-\delta)(1-\alpha)}\right)\left[\frac{1}{2} \delta(1-\beta)+\frac{1}{2}(1-\delta)(1-\alpha)\right] \\
= & h_{2}\left(\frac{\delta(1-\beta)}{\delta(1-\beta)+(1-\delta)(1-\alpha)}\right)[\delta(1-\beta)+(1-\delta)(1-\alpha)]+h_{2}\left(\frac{\delta \beta}{\delta \beta+(1-\delta) \alpha}\right)[\delta \beta+(1-\delta) \alpha]
\end{aligned}
$$

and

$$
\begin{aligned}
& H\left(Y^{(2)} \mid X, U\right) \\
= & h_{2}\left(\frac{\frac{1}{2}(1-\delta)(1-\alpha)+\frac{1}{2} \delta^{2}(1-\beta)}{\frac{1}{2}(1-\delta)(1-\alpha)+\frac{1}{2} \delta(1-\beta)}\right)\left[\frac{1}{2}(1-\delta)(1-\alpha)+\frac{1}{2} \delta(1-\beta)\right] \\
& +h_{2}\left(\frac{\frac{1}{2}(1-\delta) \alpha \delta}{\frac{1}{2}(1-\delta) \alpha+\frac{1}{2} \delta \beta}\right)\left[\frac{1}{2}(1-\delta) \alpha+\frac{1}{2} \delta \beta\right] \\
& +h_{2}\left(\frac{\frac{1}{2} \delta \beta+\frac{1}{2}(1-\delta)^{2} \alpha}{\frac{1}{2} \delta \beta+\frac{1}{2}(1-\delta) \alpha}\right)\left[\frac{1}{2} \delta \beta+\frac{1}{2}(1-\delta) \alpha\right] \\
& +h_{2}\left(\frac{\frac{1}{2}(1-\delta)(1-\beta) \delta}{\frac{1}{2} \delta(1-\beta)+\frac{1}{2}(1-\delta)(1-\alpha)}\right)\left[\frac{1}{2} \delta(1-\beta)+\frac{1}{2}(1-\delta)(1-\alpha)\right] \\
= & h_{2}\left(\frac{(1-\delta)(1-\beta) \delta}{\delta(1-\beta)+(1-\delta)(1-\alpha)}\right)[\delta(1-\beta)+(1-\delta)(1-\alpha)]+h_{2}\left(\frac{(1-\delta) \alpha \delta}{(1-\delta) \alpha+\delta \beta}\right)[(1-\delta) \alpha+\delta \beta]
\end{aligned}
$$




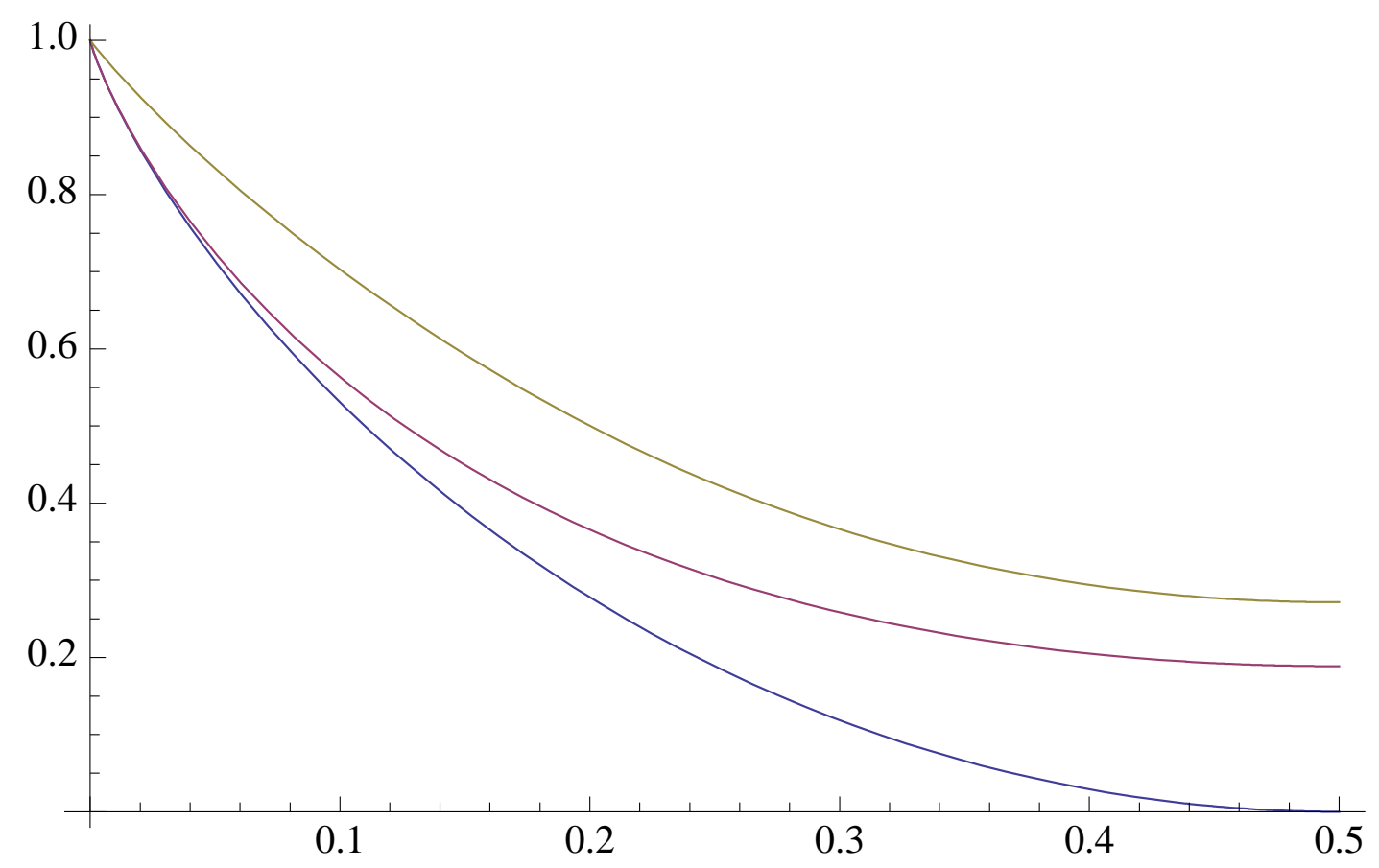

Figure 4: Capacity of the BSC with a rewrite option, where a rewrite with the same symbol has the same output as in the initial writing. The upper, middle, and lower curves correspond to the capacity for the non-causal case $C_{N C}^{C M D R W}$, for the causal case $C_{C}^{C M D R W}$, and the capacity of the standard BSC, as the channel crossover probability $\delta$ ranges in $[0,1 / 2]$.

So that:

$$
\begin{aligned}
& I\left(X, U ; Y^{(2)}\right)-I\left(U ; Y^{(1)} \mid X\right) \\
= & H\left(Y^{(2)}\right)-H\left(Y^{(1)} \mid X\right)+H\left(Y^{(1)} \mid X, U\right)-H\left(Y^{(2)} \mid X, U\right) \\
= & 1-h_{2}(\delta) \\
& +\left[h_{2}\left(\frac{\delta(1-\beta)}{\delta(1-\beta)+(1-\delta)(1-\alpha)}\right)-h_{2}\left(\frac{(1-\delta)(1-\beta) \delta}{\delta(1-\beta)+(1-\delta)(1-\alpha)}\right)\right][\delta(1-\beta)+(1-\delta)(1-\alpha)] \\
& +\left[h_{2}\left(\frac{\delta \beta}{\delta \beta+(1-\delta) \alpha}\right)-h_{2}\left(\frac{(1-\delta) \alpha \delta}{(1-\delta) \alpha+\delta \beta}\right)\right][\delta \beta+(1-\delta) \alpha] .
\end{aligned}
$$

Optimizing over $\alpha, \beta$, we obtain the capacity for the case of non-causal dependence of the channel rewrite symbols on the output symbols from the first pass:

$$
\begin{aligned}
& C_{N C}^{C M D R W} \\
= & 1-h_{2}(\delta) \\
& +\max _{0 \leq \alpha \leq 1,0 \leq \beta \leq 1}\left\{\left[h_{2}\left(\frac{\delta(1-\beta)}{\delta(1-\beta)+(1-\delta)(1-\alpha)}\right)-h_{2}\left(\frac{(1-\delta)(1-\beta) \delta}{\delta(1-\beta)+(1-\delta)(1-\alpha)}\right)\right][\delta(1-\beta)+(1-\delta)(1-\alpha)]\right. \\
& \left.+\left[h_{2}\left(\frac{\delta \beta}{\delta \beta+(1-\delta) \alpha}\right)-h_{2}\left(\frac{(1-\delta) \alpha \delta}{(1-\delta) \alpha+\delta \beta}\right)\right][\delta \beta+(1-\delta) \alpha]\right\} .
\end{aligned}
$$

Figure 4 presents plots of $C_{N C}^{C M D R W}, C_{C}^{C M D R W}$, and the capacity of the ordinary BSC, as functions of the crossover probability $\delta$. 


\section{The Gaussian Channel}

Using standard arguments, the capacity results of the previous sections can be shown to carry over to continuousalphabet channels, similarly as for the original problems of coding with transmitter state information, such as in [1]. In this section, we consider the capacity of the "writing-on-clean-paper-and-then-writing-on-its-corrupted version" channel, which has the following relations between channel inputs, channel outputs, states and actions:

$$
Y^{n}=S^{n}+X^{n}\left(M, S^{n}\right)+N^{n}=A^{n}(M)+W^{n}+X^{n}\left(M, S^{n}\right)+N^{n},
$$

where

- $S^{n}=A^{n}(M)+W^{n}$

- $W^{n}$ and $N^{n}$ are independent, $W^{n}$ is i.i.d. $\sim N\left(0, \sigma_{W}^{2}\right)$ and $N^{n}$ is i.i.d. $\sim N\left(0, \sigma_{N}^{2}\right)$

- The actions are confined to

$$
E\left[\frac{1}{n} \sum_{i=1}^{n}\left(A_{i}\right)^{2}\right] \leq P_{A}
$$

- The subsequent channel inputs are confined to

$$
E\left[\frac{1}{n} \sum_{i=1}^{n}\left(X_{i}\right)^{2}\right] \leq P_{X}
$$

The continuous-alphabet extension of Theorem 3 implies that the capacity of this channel is given by

$$
\max [I(A, U ; Y)-I(U ; S \mid A)]
$$

where the maximization is over all jointly distributed variables obeying:

- $Y=S+X+N$

- $S=A+W$

- $W \sim N\left(0, \sigma_{W}^{2}\right), N \sim N\left(0, \sigma_{N}^{2}\right), W \perp N$

- $E\left[A^{2}\right] \leq P_{A}, E\left[X^{2}\right] \leq P_{X}$

- $X$ is a function of $U, S, A$

- $U-(X, S, A)-Y$

Let $C_{G}=C_{G}\left(P_{A}, P_{X}, \sigma_{W}^{2}, \sigma_{N}^{2}\right)$ denote the maximum in (96) subject to the above constraints, and under the additional requirement that $(A, U, X, S, Y)$ be jointly Gaussian. Specifically, consider the joint distribution formed by taking:

- $A \sim N\left(0, P_{A}\right)$

- $X=\alpha A+\gamma W+G$, where $\alpha^{2} P_{A}+\gamma^{2} \sigma_{W}^{2} \leq P_{X}, G \sim N\left(0, P_{X}-\left(\alpha^{2} P_{A}+\gamma^{2} \sigma_{W}^{2}\right)\right)$

- $U=\delta X+A+\beta W$

- $W, N, A, G$ independent of each other 
It is readily verified that this joint distribution obeys the required conditions (note that $U-(X, S, A)-Y$ holds since $U$ is a function of $X, S, A$ ) and that, in fact, this is essentially (up to rescaling of variables that would not affect the expression whose maximum we seek) the most general form of the joint distribution subject to the variables being jointly Gaussian. We proceed to evaluate $I(A, U ; Y)-I(U ; S \mid A)$ for this case, and then to obtain $C_{G}$ by optimizing over $\alpha, \beta, \gamma$ and $\delta$. The variances are:

$$
\begin{aligned}
\sigma_{A}^{2} & =P_{A} \\
\sigma_{Y}^{2} & =(1+\alpha)^{2} P_{A}+(1+\gamma)^{2} \sigma_{W}^{2}+P_{X}-\left(\alpha^{2} P_{A}+\gamma^{2} \sigma_{W}^{2}\right)+\sigma_{N}^{2} \\
\sigma_{U}^{2} & =(1+\alpha \delta)^{2} P_{A}+(\gamma \delta+\beta)^{2} \sigma_{W}^{2}+\delta^{2}\left(P_{X}-\left(\alpha^{2} P_{A}+\gamma^{2} \sigma_{W}^{2}\right)\right) .
\end{aligned}
$$

The covariances are:

$$
\begin{aligned}
& E[A Y]=(1+\alpha) P_{A} \\
& E[U A]=(1+\alpha \delta) P_{A} \\
& E[U Y]=(1+\alpha)(1+\alpha \delta) P_{A}+(1+\gamma)(\beta+\gamma \delta) \sigma_{W}^{2}+\delta\left(P_{X}-\left(\alpha^{2} P_{A}+\gamma^{2} \sigma_{W}^{2}\right)\right)
\end{aligned}
$$

The conditional variances can now be computed as:

$$
\begin{aligned}
\sigma_{A \mid Y}^{2}= & \sigma_{A}^{2}-\frac{(E[A Y])^{2}}{\sigma_{Y}^{2}}=P_{A}-\frac{(1+\alpha)^{2}\left(P_{A}\right)^{2}}{(1+\alpha)^{2} P_{A}+(1+\gamma)^{2} \sigma_{W}^{2}+\left(P_{X}-\left(\alpha^{2} P_{A}+\gamma^{2} \sigma_{W}^{2}\right)\right)+\sigma_{N}^{2}}, \\
& \sigma_{U \mid A, Y}^{2}=\sigma_{U}^{2}-\frac{-2 E[A Y] E[U A] E[U Y]+(E[U Y])^{2} \sigma_{A}^{2}+(E[U A])^{2} \sigma_{Y}^{2}}{-(E[A Y])^{2}+\sigma_{A}^{2} \sigma_{Y}^{2}}
\end{aligned}
$$

and

$$
\sigma_{U \mid A, S}^{2}=\delta^{2} \sigma_{G}^{2}=\delta^{2}\left(P_{X}-\left(\alpha^{2} P_{A}+\gamma^{2} \sigma_{W}^{2}\right)\right)
$$

Thus

$$
\begin{aligned}
I(A, U ; Y)-I(U ; S \mid A) & =h(A)-h(A \mid Y)-h(U \mid A, Y)+h(U \mid A, S) \\
& =\frac{1}{2} \log \left(\frac{\sigma_{A}^{2} \sigma_{U \mid A, S}^{2}}{\sigma_{A \mid Y}^{2} \sigma_{U \mid A, Y}^{2}}\right)
\end{aligned}
$$

where $\sigma_{A}^{2}, \sigma_{U \mid A, S}^{2}, \sigma_{A \mid Y}^{2}$ and $\sigma_{U \mid A, Y}^{2}$ are given explicitly in terms of $P_{A}, P_{X}, \sigma_{W}^{2}, \sigma_{N}^{2}, \alpha, \beta, \gamma$ and $\delta$ via equations (97) through (105).

We proceed to maximize the expression in (107) with respect to $\alpha, \beta, \gamma$ and $\delta$. To this end, note first that $\sigma_{A}^{2}, \sigma_{U \mid A, S}^{2}, \sigma_{A \mid Y}^{2}$ do not depend on $\beta$. As for $\sigma_{U \mid A, Y}^{2}$, substituting the relations in equations (97) through (102) into (104) gives a quadratic expression in $\beta$ which is minimized by $\beta^{*}=\delta \frac{\alpha^{2} P_{A}-P_{X}+\gamma \sigma_{N}^{2}+\gamma^{2} \sigma_{W}^{2}}{\alpha^{2} P_{A}-P_{X}-\sigma_{N}^{2}+\gamma^{2} \sigma_{W}^{2}}$ with the value

$$
\min _{\beta} \sigma_{U \mid A, Y}^{2}=\delta^{2} \frac{\sigma_{N}^{2}\left(P_{X}-\left(\alpha^{2} P_{A}+\gamma^{2} \sigma_{W}^{2}\right)\right)}{\sigma_{N}^{2}+\left(P_{X}-\left(\alpha^{2} P_{A}+\gamma^{2} \sigma_{W}^{2}\right)\right)}
$$


Substituting the above expressions we obtain

$$
\begin{aligned}
\max _{\beta} \frac{\sigma_{A}^{2} \sigma_{U \mid A, S}^{2}}{\sigma_{A \mid Y}^{2} \sigma_{U \mid A, Y}^{2}} & =\frac{P_{A}\left(\sigma_{N}^{2}+\left(P_{X}-\left(\alpha^{2} P_{A}+\gamma^{2} \sigma_{W}^{2}\right)\right)\right)}{\sigma_{N}^{2}\left(P_{A}-\frac{(1+\alpha)^{2}\left(P_{A}\right)^{2}}{(1+\alpha)^{2} P_{A}+(1+\gamma)^{2} \sigma_{W}^{2}+\left(P_{X}-\left(\alpha^{2} P_{A}+\gamma^{2} \sigma_{W}^{2}\right)\right)+\sigma_{N}^{2}}\right)} \\
& =\frac{\left((1+2 \alpha) P_{A}+P_{X}+\sigma_{N}^{2}+(1+2 \gamma) \sigma_{W}^{2}\right)\left(\sigma_{N}^{2}+\left(P_{X}-\left(\alpha^{2} P_{A}+\gamma^{2} \sigma_{W}^{2}\right)\right)\right)}{\sigma_{N}^{2}\left(P_{X}-\alpha^{2} P_{A}+\sigma_{N}^{2}+\sigma_{W}^{2}(1+2 \gamma)\right)} \\
& =\frac{P_{A}\left(\sigma_{N}^{2}+\left(P_{X}-\left(\alpha^{2} P_{A}+\gamma^{2} \sigma_{W}^{2}\right)\right)\right)}{\sigma_{N}^{2}\left(P_{A}-\frac{(1+\alpha)^{2}\left(P_{A}\right)^{2}}{(1+\alpha)^{2} P_{A}+(1+\gamma)^{2} \sigma_{W}^{2}+\left(P_{X}-\left(\alpha^{2} P_{A}+\gamma^{2} \sigma_{W}^{2}\right)\right)+\sigma_{N}^{2}}\right)} \\
& =\frac{\left[(1+2 \alpha) P_{A}+P_{X}+1+(1+2 \gamma) \sigma_{W}^{2}\right]\left[1+\left(P_{X}-\left(\alpha^{2} P_{A}+\gamma^{2} \sigma_{W}^{2}\right)\right)\right]}{\left[P_{X}-\alpha^{2} P_{A}+1+\sigma_{W}^{2}(1+2 \gamma)\right]},
\end{aligned}
$$

where in the last line we have taken, without loss of generality, $\sigma_{N}^{2}=1$. We thus obtain

$$
\begin{aligned}
C_{G} & =C_{G}\left(P_{A}, P_{X}, \sigma_{W}^{2}, 1\right) \\
& =\frac{1}{2} \log \left(\max _{(\alpha, \gamma): \alpha^{2} P_{A}+\gamma^{2} \sigma_{W}^{2} \leq P_{X}} \frac{\left[(1+2 \alpha) P_{A}+P_{X}+1+(1+2 \gamma) \sigma_{W}^{2}\right]\left[1+\left(P_{X}-\left(\alpha^{2} P_{A}+\gamma^{2} \sigma_{W}^{2}\right)\right)\right]}{\left[P_{X}-\alpha^{2} P_{A}+1+\sigma_{W}^{2}(1+2 \gamma)\right]}\right) \cdot
\end{aligned}
$$

It is possible to find the maximizing $(\alpha, \gamma)$, and the value of the maximum in (114), in closed form. However, the expressions involved are too cumbersome to specify.

It is instructive to compare $C_{G}$ to the following lower bounds on the capacity of this channel:

- Precancelation via $X$ :

- When $P_{X} \geq \sigma_{W}^{2}$, the encoder of the sequence $X^{n}$ has access to $W^{n}$ (as it knows $S^{n}$ and $M$ and hence also $\left.A^{n}(M)\right)$, and so can use part of its power to cancel $W^{n}$, and the remaining power to amplify the action sequence $A^{n}$. The rate achieved by this strategy is

$$
\frac{1}{2} \log \left(1+\frac{\left(1+\sqrt{\frac{P_{X}-\sigma_{W}^{2}}{P_{A}}}\right)^{2} \cdot P_{A}}{\sigma_{N}^{2}}\right) .
$$

Note that this corresponds to taking $\alpha=\sqrt{\frac{P_{X}-\sigma_{W}^{2}}{P_{A}}}$ and $\gamma=-1$ in lieu of the maximum in (114).

- When $P_{X}<\sigma_{W}^{2}$, the encoder of the sequence $X^{n}$ can use all of its power to cancel as much of $W^{n}$ as it can. The encoding is done solely through the action sequence, and an effective noise power equal to $\sigma_{N}^{2}$ plus the part of $W^{n}$ that has not been canceled. The rate achieved by this strategy is

$$
\frac{1}{2} \log \left(1+\frac{P_{A}}{\left(1-\sqrt{P_{X} / \sigma_{W}^{2}}\right)^{2} \sigma_{W}^{2}+\sigma_{N}^{2}}\right) .
$$

Note that this corresponds to taking $\alpha=0$ and $\gamma=-\sqrt{P_{X} / \sigma_{W}^{2}}$.

- Both $A$ and $X$ can work together to encode for a standard AWGN channel with noise power $\sigma_{W}^{2}+\sigma_{N}^{2}$. The rate achieved is

$$
\frac{1}{2} \log \left(1+\frac{\left(1+\sqrt{P_{X} / P_{A}}\right)^{2} P_{A}}{\sigma_{W}^{2}+\sigma_{N}^{2}}\right)
$$

Note that this corresponds to taking $\alpha=\sqrt{P_{X} / P_{A}}$ and $\gamma=0$. 


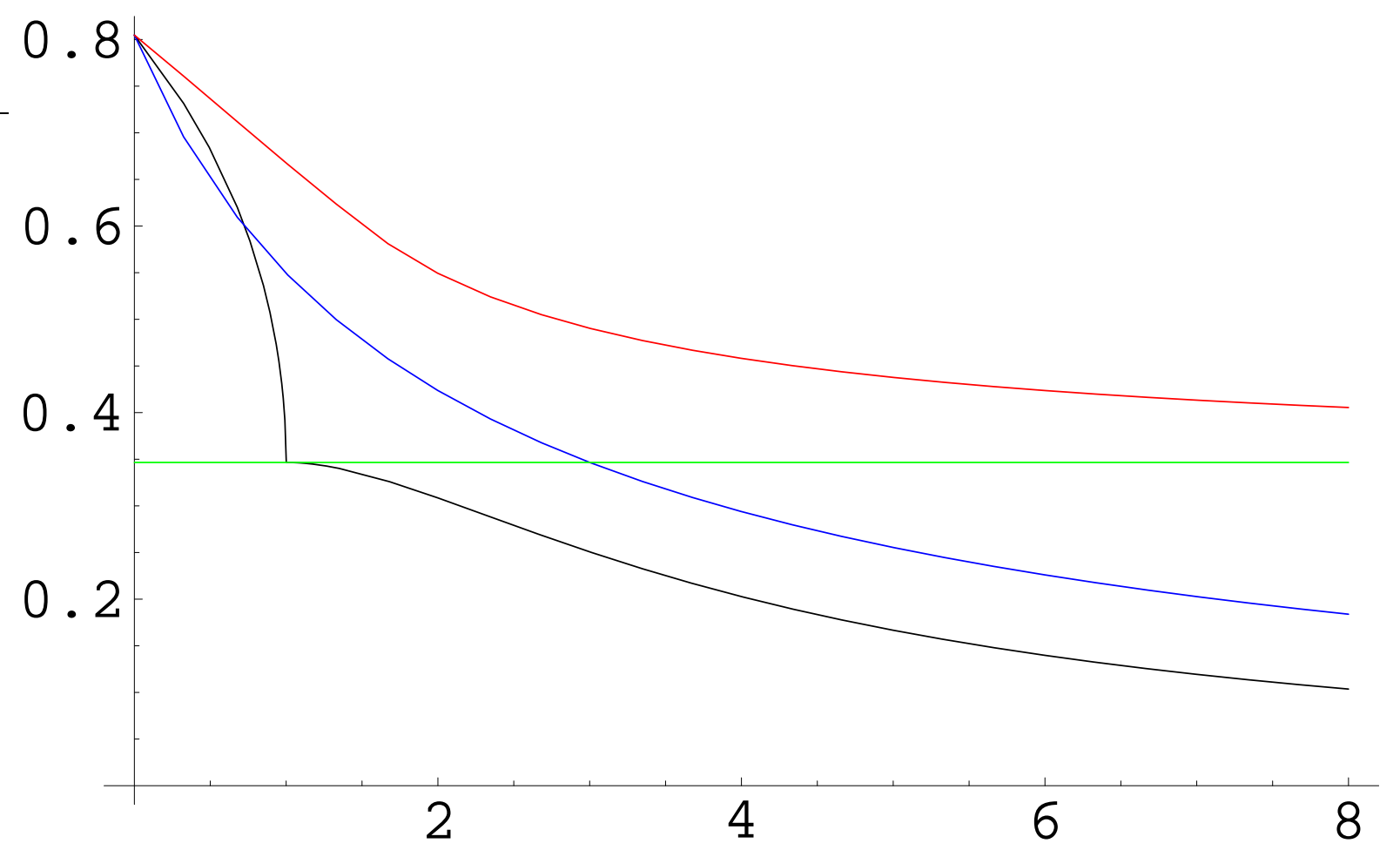

Figure 5: Lower bounds on the capacity of the Gaussian channel, for the case $P_{A}=P_{X}=\sigma_{N}^{2}=1$, as a function of $\sigma_{W}^{2}$. The red curve shows $C_{G}$. The black curve shows the cancelation bounds in (115) and (116). The blue curve shows the rate in (117), achieved when $X$ and $A$ cooperate to encode for a standard AWGN channel whose noise is $W+N$. The green line is the standard dirty paper rate in (118). As can be expected, the first three curves coincide at $\sigma_{W}^{2}=0$ and give the actual capacity for this case, which is the capacity of the AWGN channel at signal to noise ratio 4 . At the other extreme, as should be expected, the red curve is approaching the green one for large $\sigma_{W}^{2}$ since the actual capacity approaches the dirty paper capacity in the limit of large $\sigma_{W}^{2}$. For the particular case where $P_{X}=P_{A}$, dirty paper coding achieves a better rate than precancelation when $P_{X}<\sigma_{W}^{2}$ as can be seen in the graph and by comparing (116) with (118). Whether the actual capacity is given by the red curve remains to be answered.

- The rate

$$
\frac{1}{2} \log \left(1+\frac{P_{X}}{\sigma_{N}^{2}}\right)
$$

is always achievable, regardless of $\sigma_{W}^{2}$, by standard dirty paper coding (i.e., treating $S^{n}$ as interference).

Figure 5 displays a plot of $C_{G}$ as a function of $\sigma_{W}^{2}$ for the case $P_{A}=P_{X}=\sigma_{N}^{2}=1$, as well as its lower bounds, the achievable rates in (115), (116), (117) and (118). Whether $C_{G}$ is the capacity of this channel remains to be determined.

\section{Conclusions and Future Directions}

We have extended the study of channels with states known at the transmitter to the case where the formation of the states is affected by actions taken at the encoder. The fundamental limits on reliable communication for such channels were characterized. It was seen that such a framework covers channels with a 'rewrite' option based on (noiseless or noisy) feedback from the channel output in the first writing. Examples of such channels were explored in detail. 
Some questions and directions that our work leaves open for future exploration are:

- Can actions of the form $A_{i}\left(M, S^{i-1}\right)$ increase the capacity relative to actions that are not allowed to depend on past states, in the setting of Section 2, where in the encoding of the channel input $X^{n}$ non-causal dependence on the state sequence is allowed?

- For the Gaussian channel of Section 6, does a $U$ jointly Gaussian with the remaining variables achieve the maximum in (96), i.e., is $C_{G}=C$ ?

- Extension of the channel model to more than two encoding stages: In some of the motivating examples given, it makes sense to consider problems where each memory location can be rewritten into more than once. In fact, for some of the scenarios considered, particularly the one involving noisy feedback from the channel output after the first writing stage, it makes sense to consider a setting where the encoder is allowed as many 'rewrite' attempts as it desires.

\section{Acknowledgement}

Helpful discussions with Haim Permuter, Yossi Steinberg, and Sergio Verdú are acknowledged with thanks.

\section{References}

[1] M. H. M. Costa, "Writing on Dirty Paper," IEEE Trans. Inform. Theory, vol. IT-29 pp. 439-441, May 1983.

[2] T. M. Cover and J. A. Thomas. Elements of Information Theory. Wiley, New York, 1991.

[3] I. Csiszár and J. Körner. Information Theory: Coding Theorems for Discrete Memoryless Systems. Academic Press, New York, 1981.

[4] S. I. Gel'fand and M. S. Pinsker, "Coding for Channel with Random Parameters," Probl. Contr. and Inform. Theory, vol. 9, no. I, pp. 19-31, 1980.

[5] C. Heegard and A. El Gamal, "On the Capacity of Computer Memory with Defects," IEEE Trans. Inform. Theory, vol. IT-29, no. 5, pp. 731-739, September 1983.

[6] G. Keshet, Y. Steinberg and N. Merhav, "Channel Coding in the Presence of Side Information," Foundations and Trends in Communications and Information Theory, vol. 4, no. 6, 2007.

[7] A. V. Kuznetsov and B. S. Tsybakov, "Coding in a Memory with Defective Cells," Probl. Contr. and Inform. Theory, vol. 10, no. 2, pp. 52-60, 1974.

[8] N. Merhav and T. Weissman, "Coding for the feedback Gel'fand-Pinsker channel and the feedforward Wyner-Ziv source", IEEE Trans. Inform. Theory, vol. 52, no. 9, pp. 4207 - 4211, September 2006.

[9] H. Permuter and T. Weissman, "Source Coding with a Side Information 'Vending Machine' at the Decoder," submitted to ISIT 09.

[10] M. Salehi, "Cardinality Bounds on Auxiliary Variables in Multiple- User Theory via the Method of Ahlswede and Körner," Dep. Statistics, Stanford Univ., Stanford, CA, 1978, Tech. Rep. 33. 
[11] C. E. Shannon, "Channels with Side Information at the Transmitter," IBM J. Res. Dev., vol. 2, pp. 289-293, 1958.

[12] A. Somekh-Baruch, S. Shamai and S. Verdú, "Cooperative Multiple-Access Encoding with States available at One Transmitter," IEEE Trans. Inform. Theory, vol. IT-54, no. 10, pp. 4448-4469, October 2008. 